\title{
Bifurcation of Traveling Wave Solutions for (2+1)-Dimensional Nonlinear Models Generated by the Jaulent-Miodek Hierarchy
}

\author{
Yanping Ran, ${ }^{1,2}$ Jing $\mathrm{Li}^{1}{ }^{1} \mathrm{Xin} \mathrm{Li}^{1}{ }^{1}$ and Zheng $\operatorname{Tian}^{1}$ \\ ${ }^{1}$ College of Applied Science, Beijing University of Technology, Beijing 100124, China \\ ${ }^{2}$ School of Mathematics and Statistics, Tianshui Normal University, Tianshui, Gansu 741001, China \\ Correspondence should be addressed to Jing Li; leejing@bjut.edu.cn
}

Received 27 June 2014; Accepted 15 July 2014

Academic Editor: Yonghui Xia

Copyright (c) 2015 Yanping Ran et al. This is an open access article distributed under the Creative Commons Attribution License, which permits unrestricted use, distribution, and reproduction in any medium, provided the original work is properly cited.

Four (2+1)-dimensional nonlinear evolution equations, generated by the Jaulent-Miodek hierarchy, are investigated by the bifurcation method of planar dynamical systems. The bifurcation regions in different subsets of the parameters space are obtained. According to the different phase portraits in different regions, we obtain kink (antikink) wave solutions, solitary wave solutions, and periodic wave solutions for the third of these models by dynamical system method. Furthermore, the explicit exact expressions of these bounded traveling waves are obtained. All these wave solutions obtained are characterized by distinct physical structures.

\section{Introduction}

In [1-4], four (2+1)-dimensional nonlinear models generated by the Jaulent-Miodek hierarchy were developed. These nonlinear models are completely integrable evolution equations. There are many approaches to investigate nonlinear evolution equation, for example, the inverse scattering method, the Bäcklund transformation method, the Darboux transformation method, the Hirota bilinear method [1-3, 58], and the dynamical systems method [9-11]. The Hirota bilinear method [3] is used to formally derive the multiple kink solutions and multiple singular kink solutions of these models. By applying the direct symmetry method [4], group invariant solutions and some new exact solutions of the $(2+1)$-dimensional Jaulent-Miodek equation are obtained. Dynamical systems method is a very effective method to research qualitative behavior for traveling wave solutions of these completely integrable evolution equations. In [11], only considering bifurcation parametric $c$, some exact traveling wave solutions are given by applying the method of dynamical systems for these models. In this paper, all wave solutions are given by the method of dynamical systems under more general parametric conditions. Some computer symbolic systems such as Maple and Mathmatic allow us to perform complicated and tedious calculations.
Four $(2+1)$-dimensional nonlinear models generated by the Jaulent-Miodek hierarchy [3] are given by

$$
\begin{gathered}
w_{t}=-\left(w_{x x}-2 w^{3}\right)_{x}-\frac{3}{2}\left(w_{x} \partial_{x}^{-1} w_{y}+w w_{y}\right), \\
w_{t}=\frac{1}{2}\left(w_{x x}-2 w^{3}\right)_{x}-\frac{3}{2}\left(-\frac{1}{4} \partial_{x}^{-1} w_{y y}+w w_{y}\right), \\
w_{t}=\frac{1}{4}\left(w_{x x}-2 w^{3}\right)_{x}-\frac{3}{4}\left(\frac{1}{4} \partial_{x}^{-1} w_{y y}+w_{x} \partial_{x}^{-1} w_{y}\right), \\
w_{t}=2\left(w_{x x}-2 w^{3}\right)_{x}-\frac{3}{4}\left(\partial_{x}^{-1} w_{y y}-2 w_{x} \partial_{x}^{-1} w_{y}-6 w w_{y}\right),
\end{gathered}
$$

where $\partial_{x}^{-1}$ is the inverse of $\partial_{x}$ with $\partial_{x} \partial_{x}^{-1}=\partial_{x}^{-1} \partial_{x}=1$ and

$$
\partial_{x}^{-1}=\int_{-\infty}^{x} f(t) d t
$$

We will study the third model given by

$$
w_{t}=\frac{1}{4}\left(w_{x x}-2 w^{3}\right)_{x}-\frac{3}{4}\left(\frac{1}{4} \partial_{x}^{-1} w_{y y}+w_{x} \partial_{x}^{-1} w_{y}\right) .
$$

By introducing the potential

$$
w(x, y, t)=u_{x}(x, y, t)
$$


to remove the integral term in the system (3), we obtain the following equation

$$
u_{x t}+\frac{1}{4} u_{x x x x}-\frac{2}{3} u_{x}^{2} u_{x x}+\frac{3}{16} u_{y y}+\frac{3}{4} u_{x x} u_{y}=0
$$

We are interested in the wave solutions of the system (3) in this paper. Motivated by [9], we obtain dynamical properties of (11) and different wave solutions of the system (3) in detail. This paper is organized as follows. In Section 2, we establish the traveling wave equation (3) for the third model of (1). Furthermore, we obtain the first integral of dynamical governing equation of the system (11). Then, we analyze the bifurcation behaviors of the system (11). Phase portraits in the different subsets of parameter space will be presented in Section 3. In Section 4, using the information of the phase portraits in Section 3, we analyze all the possible traveling wave solutions of the system (11). Some explicit parametric representations of traveling wave solutions of (3) and the system (11) are also obtained. The final section includes brief summary, future plans, and potential fields of applications.

\section{Traveling Wave Equation for the System (3)}

We assume that the traveling wave transform of the system (3) is in the form

$$
\begin{aligned}
& u(x, y, t)=\Psi(\xi), \\
& \xi=k x+r y-c t,
\end{aligned}
$$

where $c$ is propagating wave velocity. Let $k=r, c=1$, the traveling wave transform of (6) is equivalent to $\xi=x+$ $y-c t$ [11]. So, our traveling wave transform is more general. According to physical meaning of traveling wave solutions of the system (3), we always assume that $c>0, k \neq 0$, and $r \neq 0$. Now, substituting (6) into (5), we have the traveling wave equation

$$
-k c u_{\xi \xi}+\frac{k^{4}}{4} u_{\xi \xi \xi \xi}-\frac{3}{2} k^{4} u_{\xi}^{2} u_{\xi \xi}+\frac{3}{16} r^{2} u_{\xi \xi}+\frac{3}{4} k^{2} r u_{\xi \xi} u_{\xi}=0 .
$$

Integrating (7) with respect to $\xi$ once, we have

$$
-16 k c u_{\xi}+4 k^{4} u_{\xi \xi \xi}-8 k^{4} u_{\xi}^{3}+3 r^{2} u_{\xi}+6 k^{2} r u_{\xi}^{2}=0 .
$$

Setting $u_{\xi}=\varphi$, (8) becomes

$$
-16 k c \varphi+4 k^{4} \varphi_{\xi \xi}-8 k^{4} \varphi^{3}+3 r^{2} \varphi+6 k^{2} r \varphi^{2}=0 .
$$

Furthermore, (8) can be rewritten as

$$
4 k^{4} \varphi_{\xi \xi}+\left(3 r^{2}-16 k c\right) \varphi+6 k^{2} r \varphi^{2}-8 k^{4} \varphi^{3}=0 .
$$

Letting $\varphi^{\prime}=y$, then we have the following planar system

$$
\begin{gathered}
\frac{d \varphi}{d \xi}=y, \\
\frac{d y}{d \xi}=-\frac{\left(3 r^{2}-16 k c\right)}{4 k^{4}} \varphi-\frac{3 r}{2 k^{2}} \varphi^{2}+2 \varphi^{3} .
\end{gathered}
$$

Obviously, the above system (11) is a Hamiltonian system with Hamiltonian function

$$
H(\varphi, y)=\frac{y^{2}}{2}+\frac{\left(3 r^{2}-16 k c\right)}{8 k^{4}} \varphi^{2}+\frac{r}{6 k^{2}} \varphi^{3}-\frac{\varphi^{4}}{2}
$$

In order to research the system (11), let $a=-\left(3 r^{2}-\right.$ $16 k c) / 4 k^{4}, b=-3 r / 2 k^{2}$; the system (11) becomes

$$
\begin{gathered}
\frac{d \varphi}{d \xi}=y, \\
\frac{d y}{d \xi}=a \varphi+b \varphi^{2}+2 \varphi^{3} .
\end{gathered}
$$

The Hamiltonian function of (13) is

$$
H(\varphi, y)=\frac{1}{2} y^{2}-\frac{a}{2} \varphi^{2}-\frac{1}{3} b \varphi^{3}-\frac{1}{2} \varphi^{4}
$$

\section{The Bifurcation Analysis of the System (11)}

In this section, our aim is to study the traveling wave solutions of the system (11) by applying bifurcation method and qualitative theory of dynamical systems [9, 10]. Through some special phase orbits, we obtain smooth periodic wave solutions, solitary wave solutions, kink and antikink wave solutions, and so on. Fixing $c$, we discuss the phase portrait of the system (11) along with the changes of parameters $r$ and $k$ so as to study traveling wave solutions of the system (11). Further more, through the traveling wave solutions of the system (11) and the potential relation (4), traveling wave solutions of the system (3) will be obtained.

3.1. Phase Portraits and Qualitative Analysis of the System (11). In order to investigate the phase portrait of the system (11), we set

$$
f(\varphi)=a \varphi+b \varphi^{2}+2 \varphi^{3}=\varphi\left(a+b \varphi+2 \varphi^{2}\right) .
$$

Let $\Delta=\left(33 r^{2}-128 k c\right) / 4 k^{4}$. Obviously, $f(\varphi)$ has at least one zero point $\left(\varphi_{0}, f\left(\varphi_{0}\right)\right)=(0,0)$. The number of the singular points of the system (11) may be decided by the sign of $\Delta$. Obviously, the system (11) has only one trivial singular point $(0,0)$. Thus the other singular points of the system (11) are given as follows. (1) When $\Delta<0$, the system (11) has only one trivial singular point $(0,0)$; (2) when $\Delta>0$, the system (11) has two singular points $\left(\varphi_{1,2}, 0\right)$, where $\varphi_{1}=(3 r+$ $\left.2 k^{2} \sqrt{\Delta}\right) / 8 k^{2}>\varphi_{2}=\left(3 r-2 k^{2} \sqrt{\Delta}\right) / 8 k^{2}$; (3) when $\Delta=0$, the system has a second-order singular point $\left(\varphi_{3}, 0\right)$, where $\varphi_{3}=\varphi_{1}=\varphi_{2}$.

We notice that the Jacobian of linearized system of the system (11) at the singular points is given by

$$
J\left(\varphi_{i}, 0\right)=-f^{\prime}\left(\varphi_{i}\right), \quad(i=0,1,2,3) .
$$


Thus, the characteristic values of linearized system of the system (11) at $\left(\varphi_{i}, 0\right)$ are $\lambda= \pm \sqrt{f^{\prime}\left(\varphi_{i}\right)}$. From the qualitative theory of dynamical system, we know that

(i) if $f^{\prime}\left(\varphi_{i}\right)>0,\left(\varphi_{i}, 0\right)$ is a saddle point;

(ii) if $f^{\prime}\left(\varphi_{i}\right)<0,\left(\varphi_{i}, 0\right)$ is a center point;

(iii) if $f^{\prime}\left(\varphi_{i}\right)=0,\left(\varphi_{i}, 0\right)$ is a degenerate saddle point.

Let

$$
H(\varphi, y)=h \text {, }
$$

where $h$ is Hamiltonian value. When $r^{2} \geq 4 k c$,

$$
H(\varphi, 0)=\frac{a}{2} \varphi^{2}+\frac{b}{3} \varphi^{3}+\frac{1}{2} \varphi^{4}=0
$$

has four real roots.

It is well known that the planar Hamiltonian system is determined by its potential energy level curve and its singular point in the form of $\left(\varphi^{*}, 0\right)$. So, we are interested in looking for the possible zeros of (15) and determining whether there are heteroclinic orbits, homoclinic orbits, periodic orbits at different singular points.

In order to find the heteroclinic orbits and the homoclinic orbits of the system (11), let

$$
f^{\prime}(\varphi)=a+2 b \varphi+6 \varphi^{2}=0 .
$$

From (19), we can get the following expressions of its roots:

$$
\begin{aligned}
& \varphi_{1}^{*}=\frac{3 r+\sqrt{27 r^{2}-96 k c}}{12 k^{2}}, \\
& \varphi_{2}^{*}=\frac{3 r-\sqrt{27 r^{2}-96 k c}}{12 k^{2}} .
\end{aligned}
$$

Substituting (20) into (15), we can get

$$
\begin{aligned}
f\left(\varphi_{1}^{*}\right)=- & \left(\left(3 r+\sqrt{27 r^{2}-96 k c}\right)\right. \\
& \left.\times\left(15 r^{2}-64 k c+r \sqrt{27 r^{2}-96 k c}\right)\right)\left(288 k^{6}\right)^{-1}, \\
f\left(\varphi_{2}^{*}\right)=- & \left(\left(-3 r+\sqrt{27 r^{2}-96 k c}\right)\right. \\
& \left.\times\left(-15 r^{2}-64 k c+r \sqrt{27 r^{2}-96 k c}\right)\right)\left(288 k^{6}\right)^{-1}, \\
- & f\left(\varphi_{1}^{*}\right)-f\left(\varphi_{2}^{*}\right)=-\frac{r\left(-r^{2}+4 k c\right)}{2 k^{6}} .
\end{aligned}
$$

Theorem 1. When $k>0, c>0$, from (22), one has the following.

(i) When $r= \pm 2 \sqrt{k c}$, there are two heteroclinic orbits formed by the saddle points $( \pm 2 \sqrt{k c}, 0)$.

(ii) When $r \in(-4 \sqrt{3 k c} / 3,-2 \sqrt{k c}) \cup(2 \sqrt{k c}, 4 \sqrt{3 k c} / 3)$, there are no heteroclinic orbits, while there are homoclinic orbits formed by other saddle points except for two saddle points $( \pm 2 \sqrt{k c}, 0)$.
Proof. When $r= \pm 2 \sqrt{k c}$, we have $\varphi_{0,2}=-\sqrt{k c} / k^{2}<0$ and $f^{\prime}\left(-\sqrt{k c} / k^{2}\right)<0$. According to the qualitative theory of dynamical system, $\left(\varphi_{0,2}, 0\right)$ are saddle points. Furthermore, when $r= \pm 2 \sqrt{k c},-f\left(\varphi_{1}^{*}\right)=f\left(\varphi_{2}^{*}\right)$ holds. Similarly, if $r \epsilon$ $(-4 \sqrt{3 k c} / 3,-2 \sqrt{k c}) \cup(2 \sqrt{k c}, 4 \sqrt{3 k c} / 3)$, we have that $\left(\varphi_{1,2}, 0\right)$ is the saddle point and $-f\left(\varphi_{1}^{*}\right) \neq f\left(\varphi_{2}^{*}\right)$ holds. Applying Theorems 1 and 2 [12], Theorem 1 is proved.

In order to give the details of the bifurcation, if $c>0$, $k>0$, we can obtain the following six bifurcation boundaries:

$$
\begin{gathered}
L_{1}: r=-\frac{4 \sqrt{3 k c}}{3}, \\
L_{2}: r=-2 \sqrt{k c}, \\
L_{3}: r=-\frac{8 \sqrt{66 k c}}{33}, \\
L_{4}: r=\frac{8 \sqrt{66 k c}}{33}, \\
L_{5}: r=2 \sqrt{k c}, \\
L_{6}: r=\frac{4 \sqrt{3 k c}}{3} .
\end{gathered}
$$

All these bifurcation boundaries divide the parameter space into seven regions (see Figure 1(a)) in which different phase portraits exist. All the corresponding phase portraits will be shown in Figure 2.

If $c>0, k<0$, there is one bifurcation boundary:

$$
L_{7}: r=0 \text {. }
$$

In this case, the corresponding phase portraits in two bifurcation regions $F_{7}$ and $F_{8}$ (see Figure $1(b)$ ) will be shown in Figure 3.

Assuming that the following conditions hold:

$$
k>0, \quad c>0, \quad \Delta \geq 0 .
$$

Therefore, we can obtain the phase portraits of the system (11) in Figure 2.

Set

$$
h_{i}=H\left(\varphi_{i}, y\right) \text {. }
$$

According to Figure 2, we obtain Case 1 as follows.

Case 1. Suppose that $k>0, c>0$, and $\Delta \geq 0$, in addition to one of conditions (1)-(12), we can obtain the sign of $f^{\prime}\left(\varphi_{i}\right)$ and the relation among $h\left(\varphi_{i}, 0\right)$ by choosing suitable $r, k$, and $c$, respectively.

(1) When $r<-4 \sqrt{3 k c} / 3$, the fact is that $h_{0}<h_{1}<h_{2}$ exists and the system (11) has two saddles at $\left(\varphi_{1,2}, 0\right)$ and a center at $(0,0)$ determined by $(16)$. When $h \in\left(h_{0}, h_{1}\right)$, the system (11) has a family of periodic orbits in which the periodic orbit $\Gamma_{1}$ is included (see Figure 2(a)). When $h \in$ $\left(-\infty, h_{0}\right) \cup\left(h_{2}, \infty\right)$, periodic orbits become open curves. 


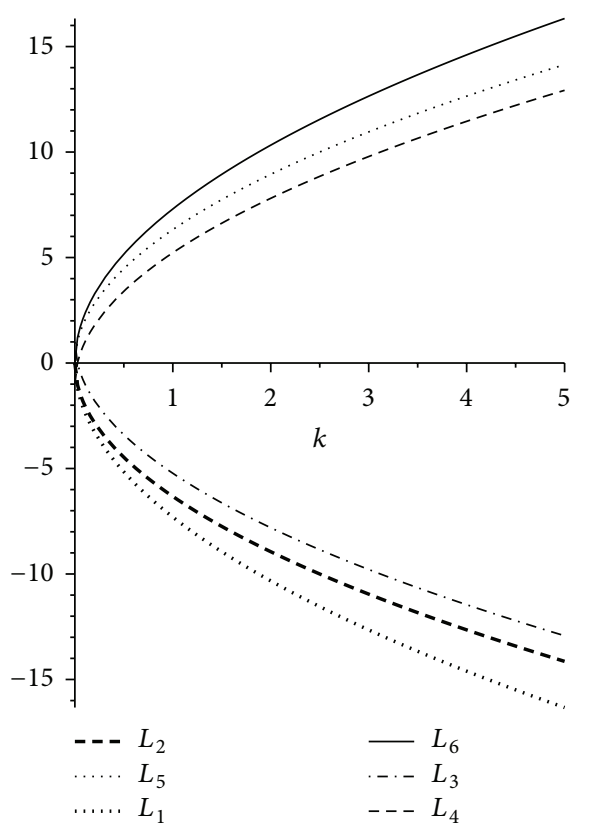

(a) $k>0, c>0, \Delta \geq 0$

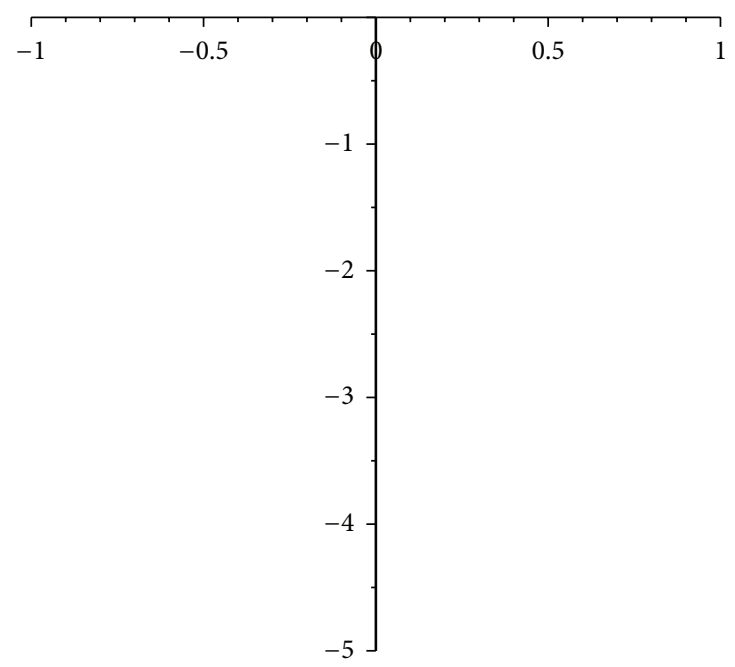

(b) $k<0, c>0$

Figure 1: Transition boundaries on $(k-r)$ plane of system (10).

(2) When $r=-4 \sqrt{3 k c} / 3$, the coefficient of $\varphi$ vanishes. Both singular points $\left(\varphi_{0,1}, 0\right)$ are degenerated to $\left(\varphi_{0}, 0\right)$ and $\left(\varphi_{2}, 0\right)$ becoming a saddle point in the system (11) (see Figure 2(b)). In the system (11), all the level curves are open.

(3) When $-4 \sqrt{3 k c} / 3<r<-2 \sqrt{k c}$, the fact is that $h_{1}<$ $h_{0}<h_{2}$ exists and the system (11) has two saddles at $\left(\varphi_{0,2}, 0\right)$ and a center at $\left(\varphi_{1}, 0\right)$. When $h=h_{0}$, the system (11) has homoclinics orbit $\Gamma_{4}$ and a special orbit $\Gamma_{5}$ (see Figure 2(c)). When $h \in\left(h_{1}, h_{0}\right)$, the system (11) has a family of periodic orbits in which the periodic orbit $\Gamma_{6}$ is included. When $h \rightarrow$ $h_{0}^{-}$, periodic orbits become the homoclinic orbit $\Gamma_{4}$. When $h \in\left(-\infty, h_{1}\right)$, periodic orbits become open curves.

(4) When $r=-2 \sqrt{k c}$, the system (11) has two saddles at $\left(\varphi_{0,2}, 0\right)$ and a center at $\left(\varphi_{1}, 0\right)$, where $\varphi_{2}=2 \varphi_{1}=-\sqrt{k c} / k^{2}$, $h_{1}=-9 c^{2} / 4 k^{4}<h_{0}=h_{2}$. When $h=h_{0}$, the system (11) has heteroclinic orbits consisting of $\Gamma_{10}$ and $\Gamma_{11}$, which connects two saddles $\left(\varphi_{0,2}, 0\right)$ (see Figure $\left.2(\mathrm{~d})\right)$. When $h \in\left(h_{1}, h_{0}\right)$, the system (11) has a family of periodic orbits in which the periodic orbit $\Gamma_{12}$ is included. But when $h \rightarrow h_{0}^{-}$, periodic orbits become the heteroclinic orbit $\Gamma_{10}$ and the orbit $\Gamma_{11}$.

(5) When $-2 \sqrt{k c}<r<-8 \sqrt{66 k c} / 33$, we can obtain $h_{2}<$ $h_{1}<h_{0}$ and the system (11) has two saddles at $\left(\varphi_{0,2}, 0\right)$ and a center at $\left(\varphi_{1}, 0\right)$ (see Figure $\left.2(\mathrm{e})\right)$. The system (11) has a family of open curves.

(6) When $r=-8 \sqrt{66 k c} / 33$, both singular points $\left(\varphi_{1,2}, 0\right)$ are degenerated to $(-2 \sqrt{66 k c} / 11,0) ;\left(\varphi_{0}, 0\right)$ becomes a saddle in the system (11) (see Figure 2(f)). The system (11) has a family of open curves.

(7) When $r=8 \sqrt{66 k c} / 33$, both singular points $\left(\varphi_{1,2}, 0\right)$ are degenerated to $(2 \sqrt{66 k c} / 11,0),\left(\varphi_{0}, 0\right)$ becomes a saddle in the system (11) (see Figure 2(g)). The system (11) has a family of open curves.

(8) When $8 \sqrt{66 k c} / 33<r<2 \sqrt{k c}$, the system (11) has two saddles at $\left(\varphi_{0,1}, 0\right)$ and a center at $\left(\varphi_{2}, 0\right)$. The system (11) has a family of open curves (see Figure $2(\mathrm{~h})$ ).

(9) When $r=2 \sqrt{k c}$, the system (11) has two saddles at $\left(\varphi_{0,2}, 0\right)$ and a center at $\left(\varphi_{1}, 0\right)$, where $\varphi_{2}=2 \varphi_{1}=-\sqrt{k c} / k^{2}$, $h_{2}=-9 c^{2} / 4 k^{4}<h_{0}=h_{1}$. When $h=h_{0}$, the system (11) has heteroclinic orbits consisting of $\Gamma_{13}$ and $\Gamma_{14}$, which connects two saddles $\left(\varphi_{0,2}, 0\right)$. When $h \in\left(h_{1}, h_{0}\right)$, the system (11) has a family of periodic orbits in which the periodic orbit $\Gamma_{15}$ is included (see Figure 2(i)). But when $h \rightarrow h_{0}^{+}$, periodic orbits become the heteroclinic orbit $\Gamma_{13}$ and the orbit $\Gamma_{14}$.

(10) When $2 \sqrt{k c}<r<4 \sqrt{3 k c} / 3$, the system (11) has two saddles at $\left(\varphi_{0,1}, 0\right)$ and a center at $\left(\varphi_{2}, 0\right)$ and $h_{2}<h_{0}<h_{1}$ exists. When $h=h_{0}$, the system (11) has homoclinics orbit $\Gamma_{7}$ and a special orbit $\Gamma_{8}$. When $h \in\left(h_{0}, h_{1}\right)$, the system (11) has a family of periodic orbits in which the periodic orbit $\Gamma_{9}$ is included (see Figure 2(j)). When $h \rightarrow h_{0}^{+}$, periodic orbits become the homoclinics orbit $\Gamma_{7}$. When $h \in\left(h_{1}, \infty\right)$, periodic orbits become open curves.

(11) When $r=4 \sqrt{3 k c} / 3$, the coefficient of $\varphi$ vanishes. Both singular points $\left(\varphi_{0,2}, 0\right)$ are degenerated to $\left(\varphi_{0}, 0\right)$ and $\left(\varphi_{1}, 0\right)$ becoming a saddle in the system (11) (see Figure $2(\mathrm{k})$ ). In the system (11), all the level curves are open.

(12) When $r>4 \sqrt{3 k c} / 3$, the system (11) has two saddles at $\left(\varphi_{1,2}, 0\right)$ and a center at $(0,0)$ and $h_{0}<h_{2}<h_{1}$ exists. When $h \in\left(h_{0}, h_{2}\right)$, the system (11) has a family of periodic orbits in which the periodic orbit $\Gamma_{16}$ is included. When $h \epsilon$ $\left(-\infty, h_{0}\right) \cup\left(h_{1}, \infty\right)$, periodic orbits become open curves (see Figure 2(l)). 


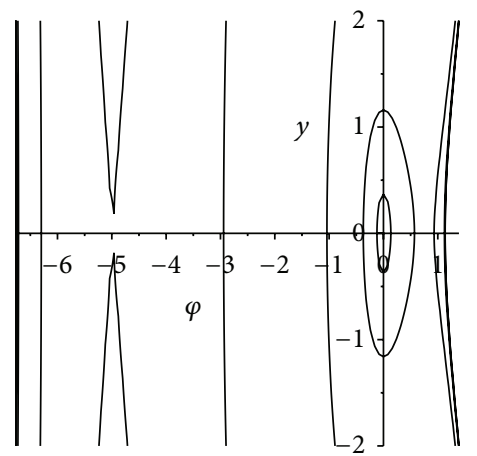

(a) $r<-4 \sqrt{3 k c} / 3$

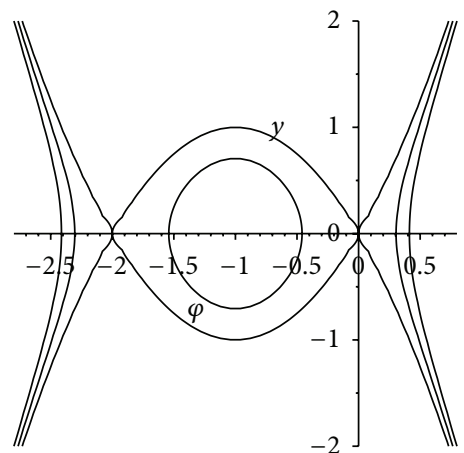

(d) $r=-2 \sqrt{k c}$

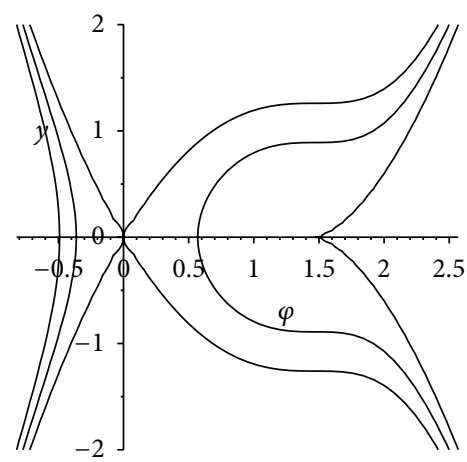

(g) $r=8 \sqrt{66 k c} / 33$

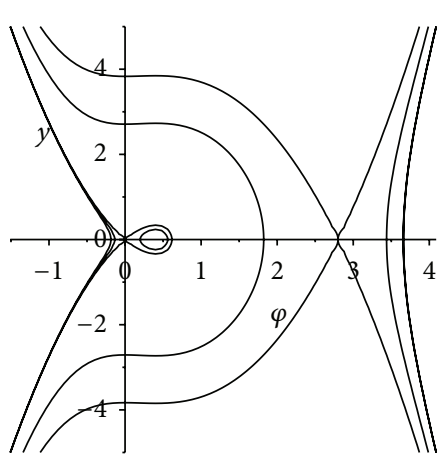

(j) $2 \sqrt{k c}<r<4 \sqrt{3 k c} / 3$

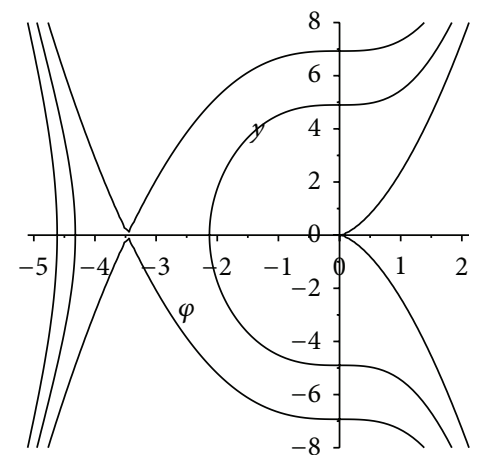

(b) $r=-4 \sqrt{3 k c} / 3$

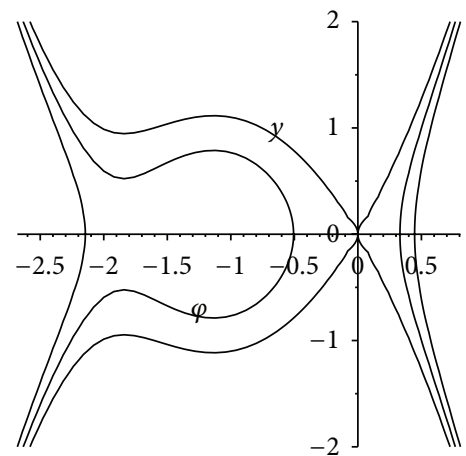

(e) $-2 \sqrt{k c}<r<-8 \sqrt{66 k c} / 33$

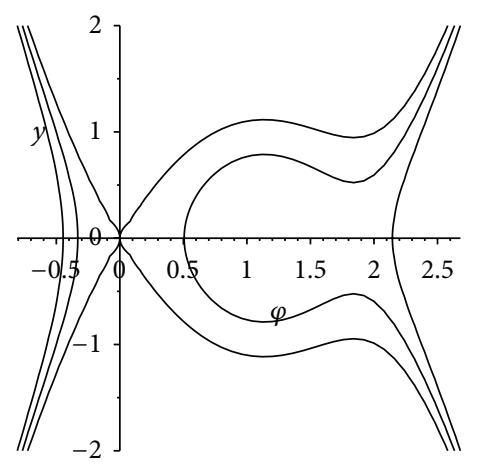

(h) $8 \sqrt{66 k c} / 33<r<2 \sqrt{k c}$

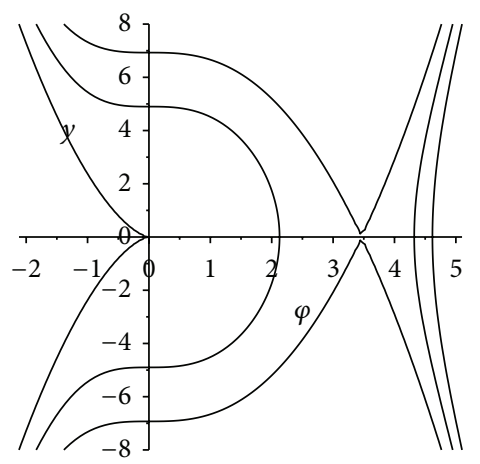

(k) $r=4 \sqrt{3 k c} / 3$

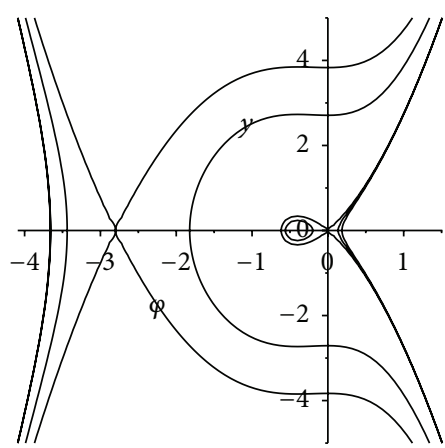

(c) $-4 \sqrt{3 k c} / 3<r<-2 \sqrt{k c}$

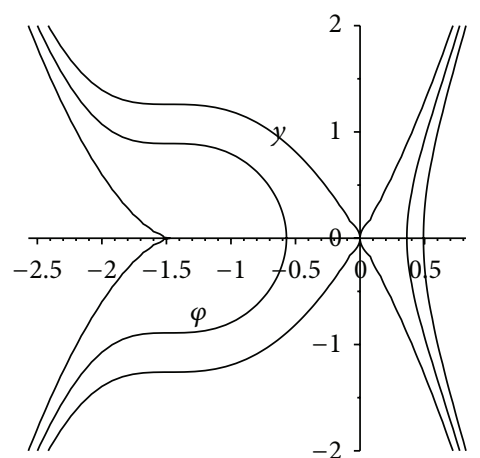

(f) $r=-8 \sqrt{66 k c} / 33$

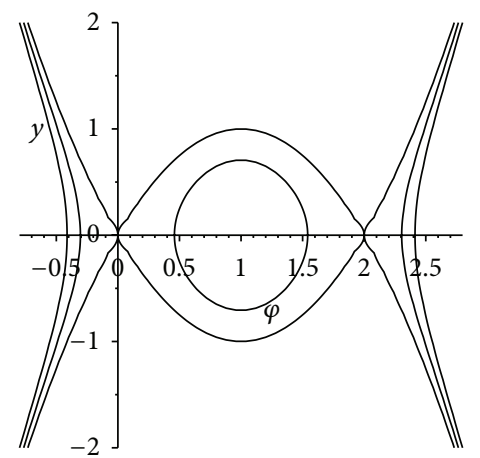

(i) $r=2 \sqrt{k c}$

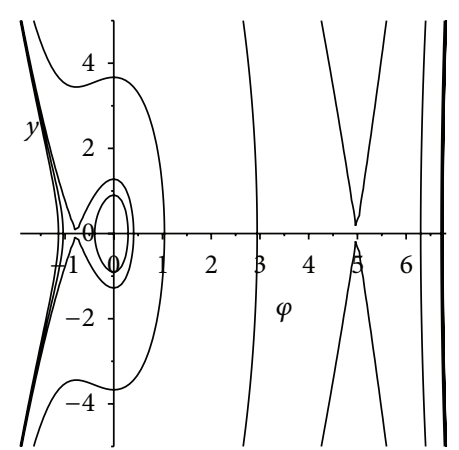

(l) $r>4 \sqrt{3 k c} / 3$

FIgURE 2: The bifurcation phase portraits in different regions of Figure 1(a) for the system (11). 


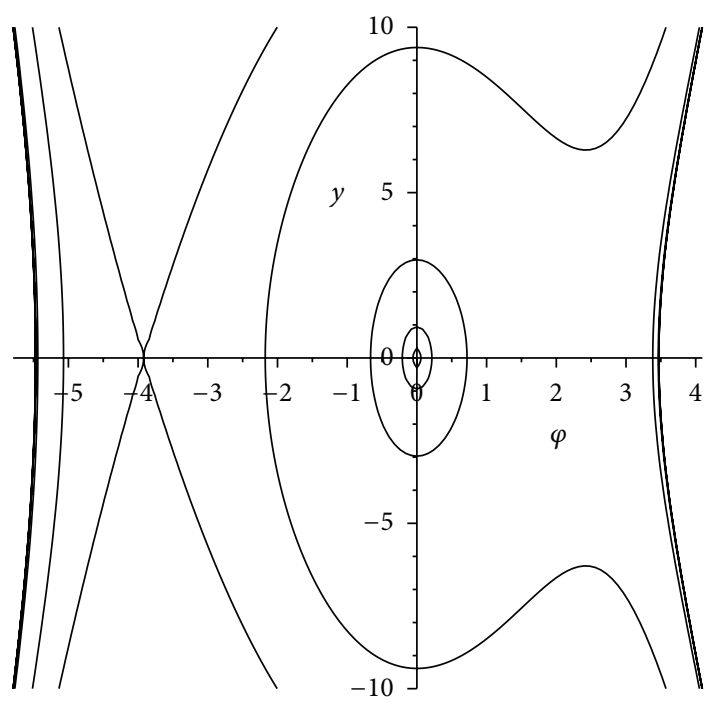

(a) $r<0$

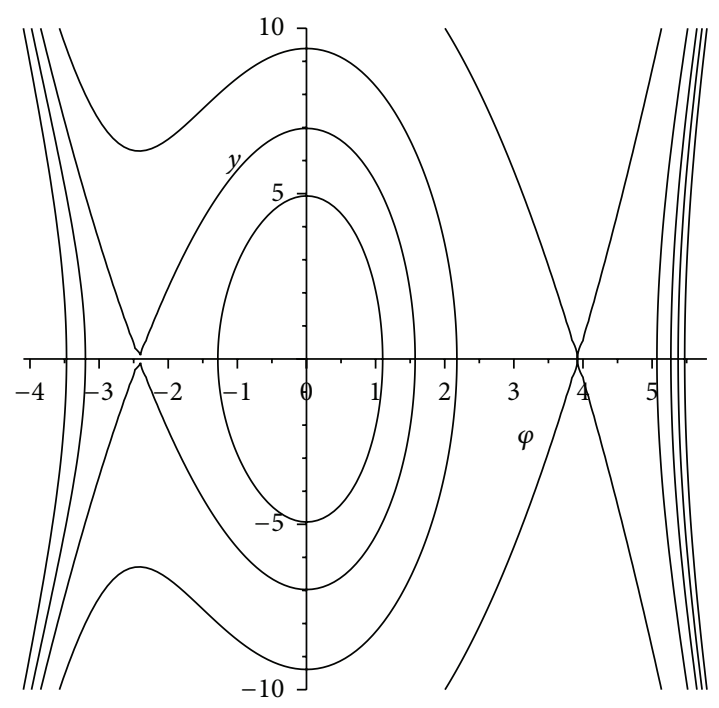

(b) $r>0$

FIGURE 3: The bifurcation phase portraits in different regions of Figure 1(b) for the system (11).

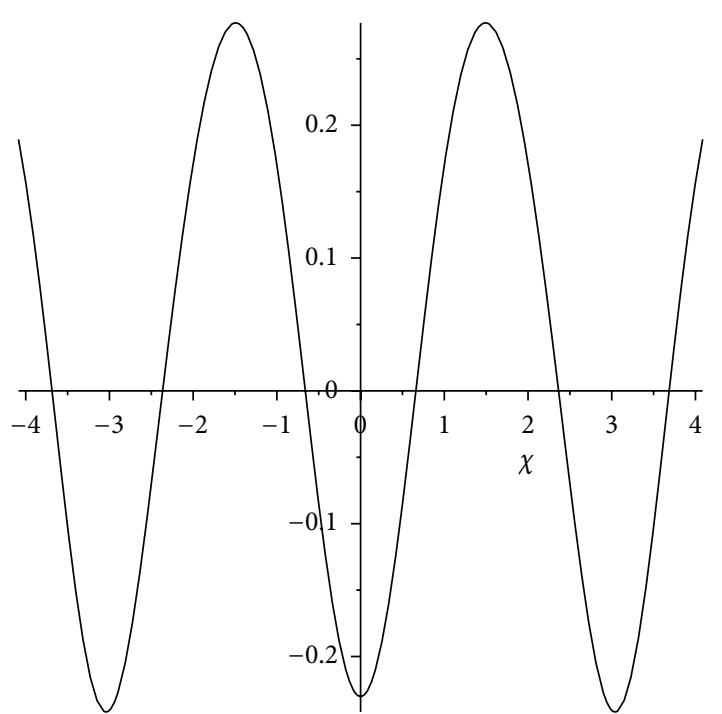

(a) $\varphi_{1}(\xi)$

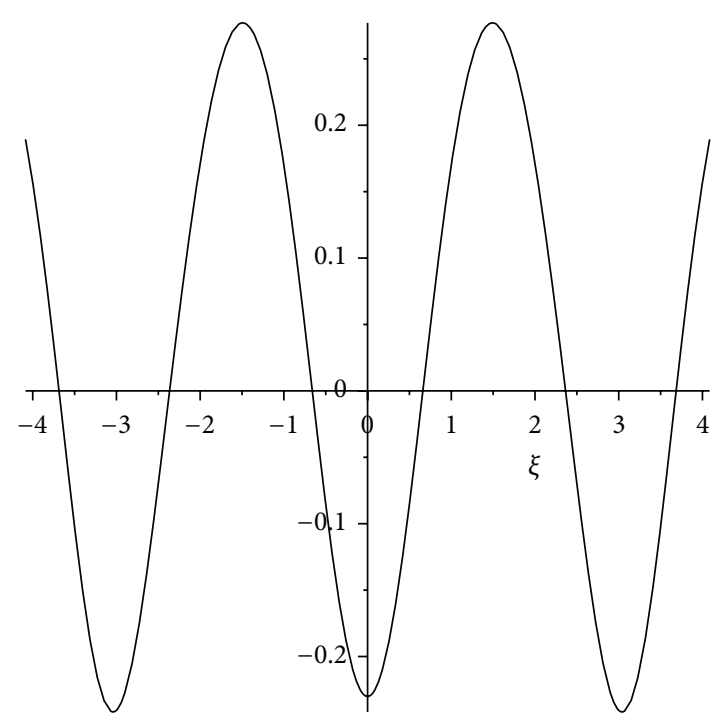

(b) $w_{1}(\xi)\left(c_{1}=0\right)$

FIGURE 4: The periodic wave solutions of the system (11) and the system (3) when $r<-4 \sqrt{3 k c} / 3, k>0, c>0$, and $\Delta \geq 0$.

Assuming that the following conditions

$$
k<0, \quad c>0
$$

hold, according to Figure 3, we obtain Case 2 as follows.

Case 2. Suppose that $k<0, c>0$, similarly, we have the following.

(13) When $r<0$, the system (11) has two saddles at $\left(\varphi_{1,2}, 0\right)$ and a center at $(0,0)$ and $h_{0}<h_{1}<h_{2}$ exists. When $h \in$ $\left(h_{0}, h_{1}\right) \cup\left(h_{1}, h_{2}\right)$, the system (11) has a family of periodic orbits in which the periodic orbit $\Gamma_{17}$ is included; under other cases, periodic orbits become open curves (see Figure 4(a)).
(14) When $r>0$, the system (11) has two saddles at $\left(\varphi_{1,2}, 0\right)$ and a center at $(0,0)$ and $h_{0}<h_{2}<h_{1}$ exists. When $h \in\left(h_{0}, h_{2}\right) \cup\left(h_{2}, h_{1}\right)$, the system (11) has a family of periodic orbits in which the periodic orbit $\Gamma_{18}$ is included; under other cases, periodic orbits become open curves (see Figure 4(b)).

\section{Smooth Solitary Wave Solutions, Periodic Wave Solutions, and Kink Wave Solutions for the System (11) and the System (3)}

In this section, we will seek all traveling wave solutions which correspond to the special bounded phase orbits of the system (11) in Section 3. The explicit expressions of the system (3) are 
also obtained by all traveling wave solutions of the system (11) and the relation (4).

4.1. Smooth Solitary Wave Solutions, Periodic Wave Solutions, and Kink and Antikink Wave Solutions of the System (11). From the qualitative theory of dynamical system, we know that a smooth solitary wave solution of a partial differential system corresponds to a smooth homoclinic orbit of a traveling wave equation. A periodic orbit of traveling wave equation corresponds to a periodic traveling wave solution of a partial differential system. Similarly, a smooth heteroclinic orbit of traveling wave equation corresponds to a smooth kink (antikink) wave solution of a partial differential system.

According to the above analysis, in this section, we consider the existence and the explicit exact expressions of smooth periodic wave solutions, smooth solitary wave solutions, and smooth kink (antikink) wave solutions for the system (11) and the system (3) under the parameter conditions $(I)$ and $(I I)$.

Firstly, we consider the existence of smooth periodic wave solutions under parameter conditions $(I)$ and $(I I)$.

Proposition 2. (i) When $k>0, c>0$, and $\Delta \geq 0$, the system (11) has a family of smooth periodic wave solutions (see Figure 2), which correspond to $H(\varphi, y)=h, h \in I$, where $I$ is one of intervals in (1), (3), (4), (9), (10), and (12) of Case 1.

(1) When $r<-4 \sqrt{3 k c} / 3, h \in\left(h_{0}, h_{1}\right)$, where $h_{0}<h_{1}<h_{2}$ in Case 1(1) (see Figure 2(a)).

(2) When $-4 \sqrt{3 k c} / 3<r<-2 \sqrt{k c}, h \in\left(h_{1}, h_{0}\right)$, where $h_{1}<h_{0}<h_{2}$ in Case 1(3) (see Figure 2(c)).

(3) When $r=-2 \sqrt{k c}, h \in\left(h_{1}, h_{0}\right)$, where $h_{1}<h_{0}=h_{2}$ in Case 1(4) (see Figure 2(d)).

(4) When $r=2 \sqrt{k c}, h \in\left(h_{2}, h_{0}\right)$, where $h_{2}<h_{0}=h_{1}$ in Case 1(9) (see Figure 2(i)).

(5) When $2 \sqrt{k c}<r<4 \sqrt{3 k c} / 3, h \in\left(h_{0}, h_{1}\right)$, where $h_{2}<$ $h_{0}<h_{1}$ in Case 1(10) (see Figure 2(j)).

(6) When $r>4 \sqrt{3 k c} / 3, h \in\left(h_{0}, h_{2}\right)$, where $h_{0}<h_{1}<h_{2}$ in Case 1(12) (see Figure 2(l)).

(ii) When $k\langle 0, c\rangle 0$, the system (11) has a family of smooth periodic wave solutions (see Figure 2), which correspond to $H(\varphi, y)=h, h \in I$, where $I$ is one of the intervals in (13) and (14) of Case 2.

(1) When $r<0, h \in\left(h_{0}, h_{1}\right) \cup\left(h_{1}, h_{2}\right)$, where $h_{0}<h_{1}<h_{2}$ in Case 2(13) (see Figure 3(a)).

(2) When $r<0, h \in\left(h_{0}, h_{2}\right) \cup\left(h_{2}, h_{1}\right)$, where $h_{0}<h_{2}<h_{1}$ in Case 2(14) (see Figure 3(b)).

Secondly, we discuss the existence of solitary wave solutions under group (I). We can summarize the results for the system (11) from Figures 2(c) and 2(j).
Proposition 3. Under conditions (I), one has following results.

(i) When $-4 \sqrt{3 k c} / 3<r<-2 \sqrt{k c}$, the system (11) has a smooth solitary wave solution of valley type, which corresponds to the orbit $\Gamma_{4}$ of $H(\varphi, y)=h_{0}$.

(ii) When $2 \sqrt{k c}<r<4 \sqrt{3 k c} / 3$, the system (11) has a smooth solitary wave solution of peak type, which corresponds to the orbit $\Gamma_{19}$ of $H(\varphi, y)=h_{0}$.

Finally, we mention the conditions of existence for kink wave solutions of the system (11).

Proposition 4. When conditions (I) hold, the system (11) has smooth kink (antkink) under one of the following conditions:

(1) $r=-2 \sqrt{k c}$, the system (11) has smooth kink which corresponds to the orbits $\Gamma_{9}$ and $\Gamma_{10}$ of $H(\varphi, y)=h_{0}$ (see Figure 2(d));

(2) $r=2 \sqrt{k c}$, the system (11) has smooth kink which corresponds to the orbits $\Gamma_{14}$ and $\Gamma_{15}$ of $H(\varphi, y)=h_{0}$ (see Figure 2(i)).

4.2. Exact Traveling Wave Solutions of the System (11) and the System (3). Firstly, we will obtain some explicit expressions of traveling wave solutions for the system (11) when conditions (I) and (II) hold. Furthermore, using potential (4) for the system (3), its exact traveling wave solutions are given as follows.

We only choose one of the periodic orbits to calculate periodic wave solutions.

(1) Periodic wave solutions for the system (11) and the system (3).

There are periodic orbits such as $\Gamma_{1}, \Gamma_{6}, \Gamma_{9}, \Gamma_{12}, \Gamma_{15}, \Gamma_{16}$, $\Gamma_{17}$, and $\Gamma_{18}$ (see Figures 2(a), 2(c), 2(d), 2(i), 2(j), 2(l), 3(a), and $3(\mathrm{~b}))$, which correspond to periodic wave solutions for the system (11). We only choose one of the periodic orbits (see Figure 2(a)) to calculate periodic wave solutions. This method can be used for other periodic orbits.

When $r<-4 \sqrt{3 k c} / 3$ (see Figure 2(a)), we notice that there are periodic orbit $\Gamma_{1}$ and two special orbits $\Gamma_{2}, \Gamma_{3}$ passing the points $\left(\varphi_{4}, 0\right),\left(\varphi_{5}, 0\right),\left(\varphi_{6}, 0\right)$, and $\left(\varphi_{7}, 0\right)$. In the $(\varphi, y)$ plane the expressions of the orbits are given as

$$
\begin{aligned}
y & = \pm \sqrt{-\frac{\left(3 r^{2}-16 k c\right)}{8 k^{4}} \varphi^{2}-\frac{27 r}{k^{2}} \varphi^{3}+\varphi^{4}+h} \\
& = \pm \sqrt{\left(\varphi-\varphi_{4}\right)\left(\varphi-\varphi_{5}\right)\left(\varphi-\varphi_{6}\right)\left(\varphi-\varphi_{7}\right)},
\end{aligned}
$$

where $\varphi_{4}<\varphi_{5}<0<\varphi_{6}<\varphi_{7}$.

Substituting (26) into $d \varphi / d \xi=y$ and integrating them along $\Gamma_{1}, \Gamma_{2}$, and $\Gamma_{3}$, it follows that

$$
\pm \int_{\varphi_{5}}^{\varphi} \frac{1}{\sqrt{\left(\varphi-\varphi_{4}\right)\left(\varphi-\varphi_{5}\right)\left(\varphi-\varphi_{6}\right)\left(\varphi-\varphi_{7}\right)}} d s=\int_{0}^{\xi} d s
$$


Completing the above integral, we obtain one of the periodic traveling wave solutions (see Figure 4(a)) of (26):

$$
\begin{aligned}
& \varphi_{1}(\xi) \\
& =\varphi_{4}+\frac{\left(\varphi_{5}-\varphi_{4}\right) \operatorname{sn}\left(\xi, \varphi_{4}, \varphi_{5}, \varphi_{6}, \varphi_{7}\right)}{1-\left(\left(\varphi_{6}-\varphi_{5}\right) /\left(\varphi_{6}-\varphi_{4}\right)\right) \operatorname{sn}\left(\xi, \varphi_{4}, \varphi_{5}, \varphi_{6}, \varphi_{7}\right)},
\end{aligned}
$$

where $\operatorname{sn}\left(\xi, \varphi_{4}, \varphi_{5}, \varphi_{6}, \varphi_{7}\right)=s n^{2}\left(\sqrt{2\left(\varphi_{5}-\varphi_{7}\right)\left(\varphi_{4}-\varphi_{6}\right)} \xi / 2\right.$, $\left.\left(\varphi_{6}-\varphi_{5}\right)\left(\varphi_{7}-\varphi_{4}\right) /\left(\varphi_{7}-\varphi_{5}\right)\left(\varphi_{6}-\varphi_{4}\right)\right)$.

Noting (6), we obtain the the following exact periodic wave solutions of the system (11) from (28):

$$
\begin{aligned}
& q_{1}(x, y, t) \\
& =\varphi_{4}+\frac{\left(\varphi_{5}-\varphi_{4}\right) \operatorname{sn}\left(x, y, t, \varphi_{4}, \varphi_{5}, \varphi_{6}, \varphi_{7}\right)}{1-\left(\left(\varphi_{6}-\varphi_{5}\right) /\left(\varphi_{6}-\varphi_{4}\right)\right) \operatorname{sn}\left(x, y, t, \varphi_{4}, \varphi_{5}, \varphi_{6}, \varphi_{7}\right)},
\end{aligned}
$$

where $s n\left(x, y, t, \varphi_{4}, \varphi_{5}, \varphi_{6}, \varphi_{7}\right)=s n^{2}\left(2 \sqrt{\left(\varphi_{5}-\varphi_{7}\right)\left(\varphi_{4}-\varphi_{6}\right)}(k x+\right.$ $\left.r y-c t) / 2,\left(\varphi_{6}-\varphi_{5}\right)\left(\varphi_{7}-\varphi_{4}\right) /\left(\varphi_{7}-\varphi_{5}\right)\left(\varphi_{6}-\varphi_{4}\right)\right) . q_{1}(x, y, t)$ is one of the smooth periodic wave solutions of the system (11).

Since $u_{1}(\xi)=\int \varphi_{1}(\xi) d \xi$, integrating (28) about $\xi$, by (6), we can obtain one of smooth wave solutions $u_{1}(x, y, t)$ of system (5). Applying the potential (4), the periodic wave solution for the system (3) is obtained as follows:

$$
w_{1}(x, y, t)=k q_{1}(x, y, t)+c_{1},
$$

where $c_{1}$ is a constant.

Noting (6), the periodic traveling wave solution $w_{1}(\xi)$ for the system (3) is obtained (see Figure 4(b)).

Remark 5. In [11], some periodic wave solutions of the system (11) are obtained, but the periodic wave solutions of the system (3) are not given. The periodic wave solutions of the system (3) cannot be derived by the method [3]. In this paper, we obtain all periodic wave solutions of the system (11) and the system (3).

(2) Solitary wave solutions for the system (11) and the system (3).

When $-4 \sqrt{3 k c} / 3<r<-2 \sqrt{k c}$, from the phase portrait (see Figure 2(c)), we notice that there are a homoclinic orbit $\Gamma_{4}$ and a special orbit $\Gamma_{5}$ passing the points $\left(\varphi_{8}, 0\right),\left(\varphi_{9}, 0\right)$, and $(0,0)$. In $(\varphi, y)$-plane, the expressions of the orbits are given as

$$
y= \pm \sqrt{\varphi^{2}\left(\varphi-\varphi_{8}\right)\left(\varphi-\varphi_{9}\right)}
$$

when $H(\varphi, y)=0$, where $\varphi_{8}=\left(r-2 \sqrt{r^{2}-4 k c}\right) / 2 k^{2}<\varphi_{9}=$ $\left(r+2 \sqrt{r^{2}-4 k c}\right) / 2 k^{2}<0$.

Substituting (31) into $d \varphi / d \xi=y$ and integrating them along $\Gamma_{4}$ and $\Gamma_{5}$, we have

$$
\pm \int_{\varphi_{9}}^{\varphi} \frac{1}{\sqrt{\varphi^{2}\left(\varphi-\varphi_{8}\right)\left(\varphi-\varphi_{9}\right)}} d s=\int_{0}^{\xi} d s .
$$

Completing the above integral, we obtain the following solitary wave solution (see Figure 5(a)) of the system (11):

$$
\varphi_{2}(\xi)=\frac{2 \varphi_{8} \varphi_{9}}{\left(\varphi_{8}-\varphi_{9}\right) \cosh \left((\sqrt{2} / 2) \sqrt{\varphi_{8} \varphi_{9}} \xi\right)+\varphi_{8}+\varphi_{9}} .
$$

Noting (6), we obtain the following exact solitary wave solutions (see Figure 5(a)) of the system (11) from (33):

$$
\begin{aligned}
& q_{2}(x, y, t) \\
& \quad=-\frac{2 a k^{2}}{2 \sqrt{r^{2}-4 k c} \cosh ((\sqrt{2 a} / 2)(k x+r y-c t))-r},
\end{aligned}
$$

where $q_{2}(x, y, t)$ is a solitary wave solution of the system (11).

Since $u(\xi)=\int \varphi(\xi) d \xi$, integrating (33) about $\xi$, we can obtain

$$
\begin{aligned}
& u_{2}(\xi) \\
& \quad=2 \sqrt{2} \tanh ^{-1}\left(\frac{\left(r+2 \sqrt{r^{2}-4 k c}\right) \tanh (\sqrt{2 a} \xi / 4)}{2 k^{2} \sqrt{a}}\right) .
\end{aligned}
$$

According to (6), wave solutions of traveling wave equation (7) from (5) are able to obtain

$$
\begin{aligned}
& u_{2}(x, y, t) \\
& =2 \sqrt{2} \tanh ^{-1}( \\
& \quad\left(r+2 \sqrt{r^{2}-4 k c}\right)\left(\frac{\sqrt{2 a}(k x+r y-c t)}{4}\right) \\
& \times \tanh \left(\frac{\sqrt{2 a}(k x+r y-c t)}{4}\right) \\
& \left.\times\left(2 k^{2} \sqrt{a}\right)^{-1}\right),
\end{aligned}
$$

where $u_{2}(x, y, t)$ is one of the smooth wave solutions of (5).

We substitute (36) into the potential $w(x, y, t)=u_{x}(x, y, t)$ as defined in (4) to obtain

$$
\begin{aligned}
w_{2}(x, y, t)=- & \left(2 k^{3} a\left(r+2 \sqrt{r^{2}-4 k c}\right)\right) \\
\times & \left(\left(5 r^{2}+4 r \sqrt{r^{2}-4 k c}-4 k^{4} a-16 k c\right)\right. \\
& \times \cosh \left(\frac{\sqrt{2 a}(k x+r y-c t)}{4}\right)^{2} \\
& \left.-4 r \sqrt{r^{2}-4 k c}+5 r^{2}+16 k c\right)^{-1},
\end{aligned}
$$

where $w_{2}(x, y, t)$ is a solitary wave solution of the system (3). Using traveling wave transform (6), the solitary wave solution $w_{2}(\xi)$ of the system (3) is obtained (see Figure 5(b)). 


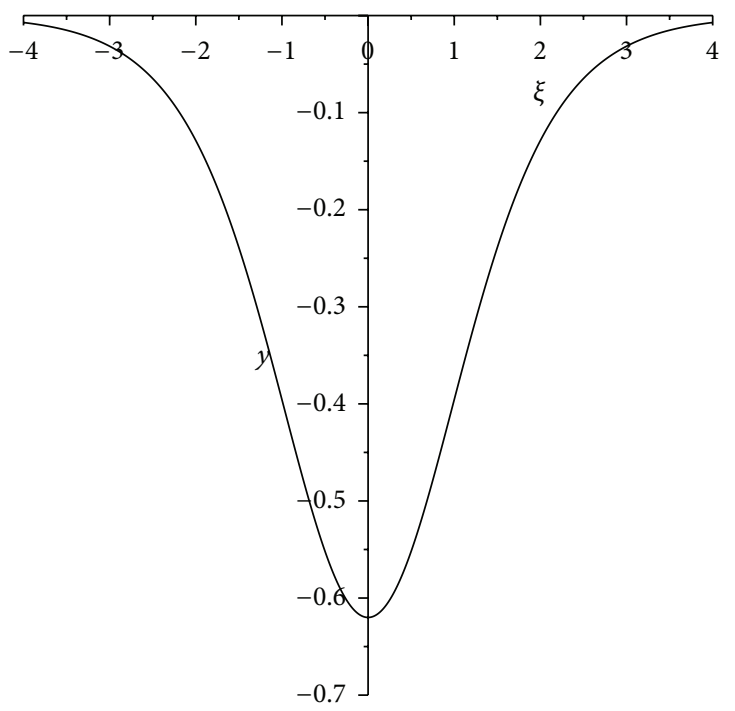

(a) $\varphi_{2}(\xi)$

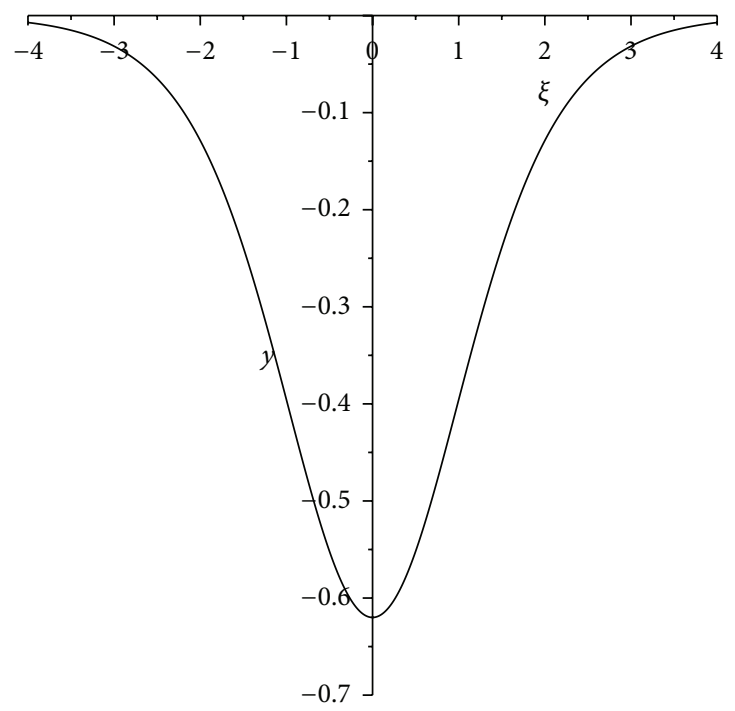

(b) $w_{2}(\xi)$

FIGURE 5: The solitary wave of the system (11) and the system (3) when $-4 \sqrt{3 k c} / 3<r<-2 \sqrt{k c}, k>0, c>0$, and $\Delta \geq 0$.

When $2 \sqrt{k c}<r<4 \sqrt{3 k c} / 3$ (see Figure $2(\mathrm{j})$ ), the expressions of the homoclinic orbit $\Gamma_{7}$ and the special orbit $\Gamma_{8}$ passing the points $\left(\varphi_{11}, 0\right),(0,0)$, and $\left(\varphi_{12}, 0\right)$ are given as in $(\varphi, y)$-plane:

$$
y= \pm \sqrt{\varphi^{2}\left(\varphi-\varphi_{11}\right)\left(\varphi-\varphi_{12}\right)},
$$

when $H(\varphi, y)=0$, where $0<\varphi_{11}=\left(r-2 \sqrt{r^{2}-4 k c}\right) / 2 k^{2}<$ $\varphi_{12}=\left(r+2 \sqrt{r^{2}-4 k c}\right) / 2 k^{2}$.

Substituting (38) into $d \varphi / d \xi=y$ and integrating them along $\Gamma_{19}$ and $\Gamma_{20}$, we have

$$
\pm \int_{\varphi}^{\varphi_{11}} \frac{1}{\sqrt{\varphi^{2}\left(\varphi-\varphi_{11}\right)\left(\varphi-\varphi_{12}\right)}} d s=\int_{0}^{\xi} d s .
$$

Completing the above integral, we obtain the following solitary wave solution (see Figure 6(a)) of the system (11):

$$
\varphi_{3}(\xi)=\frac{2 \varphi_{8} \varphi_{9}}{\left(\varphi_{9}-\varphi_{8}\right) \cosh \left((\sqrt{2} / 2) \sqrt{\varphi_{8} \varphi_{9}} \xi\right)+\varphi_{8}+\varphi_{9}} .
$$

Noting (6), we obtain the following exact wave solutions of the system (11) from (40)

$$
\begin{aligned}
q_{3} & (x, y, t) \\
& =\frac{2 k^{2} a}{2 \sqrt{r^{2}-4 k c} \cosh ((\sqrt{2 a} / 2)(k x+r y-c t))+r},
\end{aligned}
$$

where $q_{3}(x, y, t)$ is a solitary wave solution of the system (11).

Since $u(\xi)=\int \varphi(\xi) d \xi$, integrating (40) about $\xi$, we can obtain

$u_{3}(\xi)=-2 \sqrt{2} \tanh ^{-1}\left(\frac{\left(-r+2 \sqrt{r^{2}-4 k c}\right) \tanh (\sqrt{2 a} \xi / 4)}{2 k^{2} \sqrt{a}}\right)$.
According to (6), one of the smooth wave solutions of (5) is able to obtain

$$
\begin{aligned}
& u_{3}(x, y, t) \\
& =-2 \sqrt{2} \tanh ^{-1}(( \\
& \left.\quad \times \tanh \left(\frac{\sqrt{2 a}(k x+r y-c t)}{4}\right)\right) \\
& \left.\times\left(2 k^{2} \sqrt{a}\right)^{-1}\right) .
\end{aligned}
$$

Substitute (42) into the potential $w(x, y, t)=u_{x}(x, y, t)$ as defined in (4) to obtain

$$
\begin{aligned}
w_{3}(x, y, t)=( & \left.2 k^{3} a\left(r-2 \sqrt{r^{2}-4 k c}\right)\right) \\
\times & \left(\left(-5 r^{2}+4 r \sqrt{r^{2}-4 k c}+4 k^{2} a+16 k c\right)\right. \\
& \times\left(\cosh \left(\frac{\sqrt{2 a}(k x+r y-c t)}{4}\right)\right)^{2} \\
& \left.-4 r \sqrt{r^{2}-4 k c}+5 r^{2}-16 k c\right)^{-1},
\end{aligned}
$$

where $w_{3}(x, y, t)$ is a solitary wave solution of the system (3). Applying (6), the solitary wave solution $w_{3}(\xi)$ is obtained for the system (3) (see Figure 6(b)). 


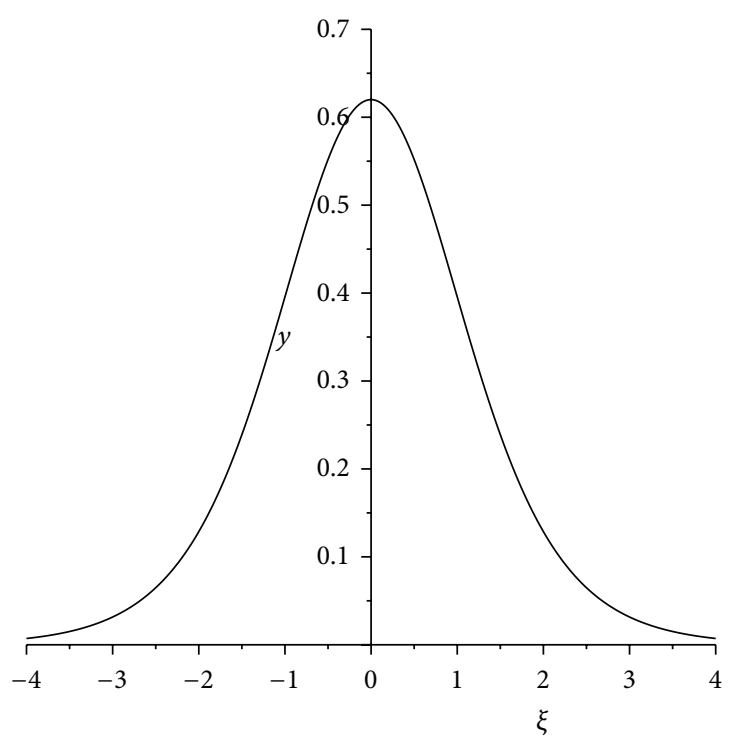

(a) $\varphi_{3}(\xi)$

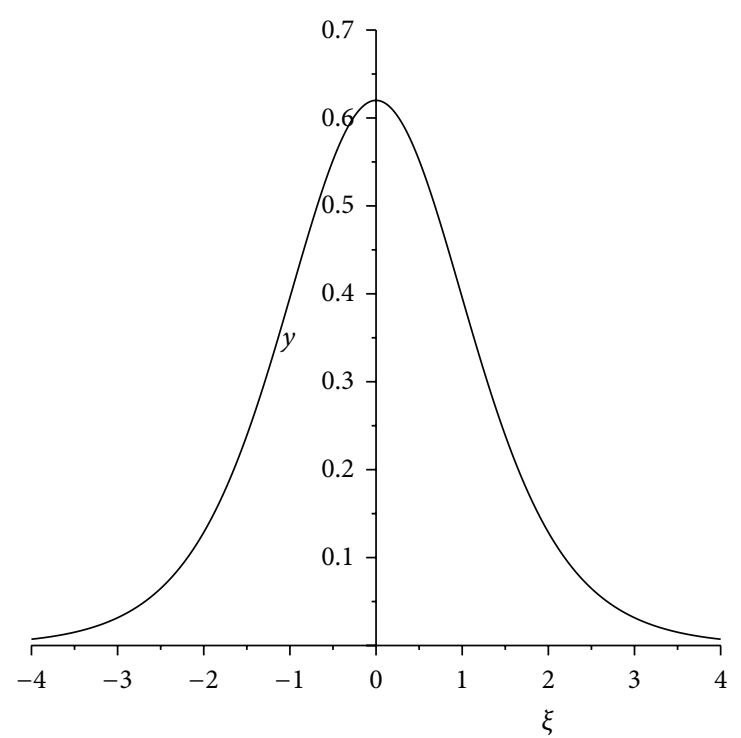

(b) $w_{3}(\xi)$

FIGURE 6: The solitary wave of the system (11) and the system (3) when $2 \sqrt{k c}<r<4 \sqrt{3 k c} / 3, k>0, c>0$, and $\Delta \geq 0$.

Remark 6. In [11], only one solitary wave solution of peak type wave solutions of the system (3) is obtained. However, the solitary wave solutions of system (3) cannot be found by Hirota's bilinear method [3]. Fortunately, we obtain all solitary wave solutions of the system (11) and the system (3).

(3) Kink (antikink) wave solutions for the system (11) and the system (3).

When $r=-2 \sqrt{k c}$ (see Figure 2(d)), in $(\varphi, y)$-plane, the expressions of the heteroclinic orbits $\Gamma_{10}$ and $\Gamma_{11}$ passing the points $\left(\varphi_{13}, 0\right),(0,0)$ are given as

$$
y= \pm \sqrt{\frac{c \varphi^{2}}{k^{3}}+2 \frac{\sqrt{k c}}{k^{2}} \varphi^{3}+\frac{1}{2} \varphi^{4}}
$$

when $H(\varphi, y)=0$, where $\varphi_{13}=-\sqrt{k c} / k^{2}<0$.

Substituting (45) into $d \varphi / d \xi=y$ and integrating them along $\Gamma_{10}$ and $\Gamma_{11}$, we have

$$
\begin{gathered}
\int_{\varphi_{13}}^{\varphi} \frac{1}{\sqrt{\varphi^{2}\left(\varphi+\sqrt{k c} / k^{2}\right)^{2}}} d s=\frac{\sqrt{2}}{2} \int_{0}^{\xi} d s, \\
-\int_{\varphi_{13}}^{\varphi} \frac{1}{\sqrt{\varphi^{2}\left(\varphi+\sqrt{k c} / k^{2}\right)^{2}}} d s=\frac{\sqrt{2}}{2} \int_{0}^{\xi} d s .
\end{gathered}
$$

Completing the above integral (46a), we obtain the following kink wave solution of the system (11) (see Figure 7(a)):

$$
\varphi_{4}(\xi)=-\frac{\sqrt{k c}\left(1-\tanh \left(\sqrt{2 k c} \xi / 2 k^{2}\right)\right)}{2 k^{2}} .
$$

Noting (6), we obtain the exact wave solution of the system (11) from (47). Consider

$$
q_{4}(x, y, t)=-\frac{\sqrt{k c}\left(1-\tanh \left(\sqrt{2 k c}(k x+r y-c t) / 2 k^{2}\right)\right)}{2 k^{2}},
$$

where $q_{4}(x, y, t)$ is a kink wave solution of the system (11).

Since $u(\xi)=\int \varphi(\xi) d \xi$, integrating (47) about $\xi$, we can obtain

$$
\begin{aligned}
& u_{4}(\xi) \\
& =-\left(\frac{1}{2 k^{2}} \sqrt{k c} \xi+\frac{\sqrt{2}}{4}\right. \\
& \quad \times\left(\ln \left(1+\tanh \left(\frac{\sqrt{2 k c} \xi}{2 k^{2}}\right)\right)\right. \\
& \left.\left.\quad+\frac{\sqrt{2}}{4} \ln \left(1-\tanh \left(\frac{\sqrt{2 k c} \xi}{2 k^{2}}\right)\right)\right)\right) .
\end{aligned}
$$

According to (6), kink wave solutions of (5) are able to obtain $u_{4}(x, y, t)$

$$
\begin{aligned}
=-\left(\frac{1}{2 k^{2}} \sqrt{k c}\right. & (k x+r y-c t) \\
+\frac{\sqrt{2}}{4}( & \ln \left(-\tanh \left(\frac{\sqrt{2 k c}(k x+r y-c t)}{2 k^{2}}\right)-1\right) \\
+ & \left.\left.\frac{\sqrt{2}}{4} \ln \left(1-\tanh \left(\frac{\sqrt{2 k c}(k x+r y-c t)}{2 k^{2}}\right)\right)\right)\right) .
\end{aligned}
$$




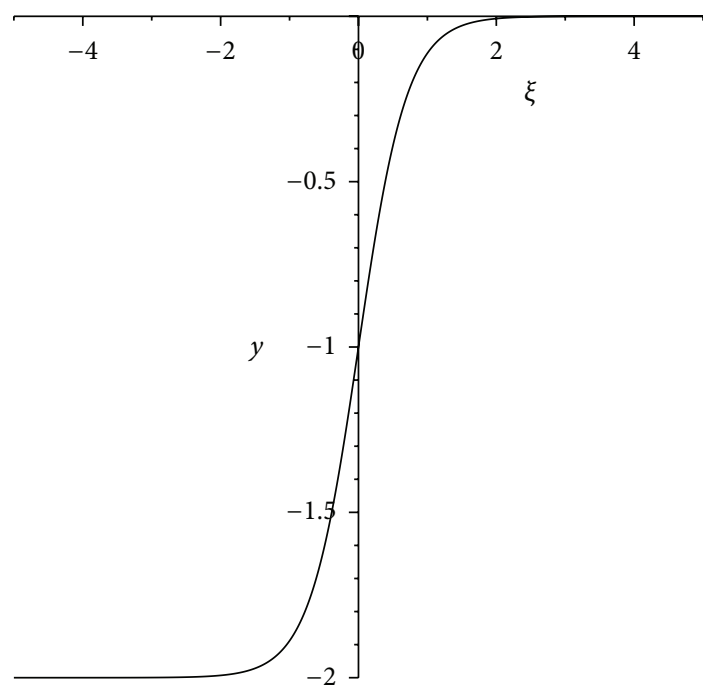

(a) $\varphi_{4}(\xi)=-\left(\sqrt{k c}\left(1-\tanh \left(\sqrt{2 k c} \xi / 2 k^{2}\right)\right)\right) / 2 k^{2}$

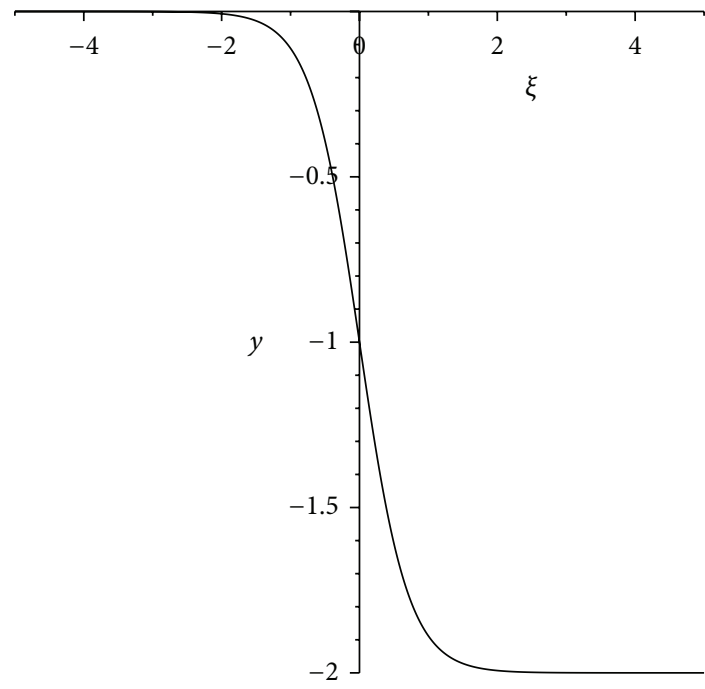

(c) $\varphi_{4^{\prime}}(\xi)=\sqrt{k c}\left(1+\tanh \left(\sqrt{2 k c} \xi / 2 k^{2}\right)\right) /-2 k^{2}$

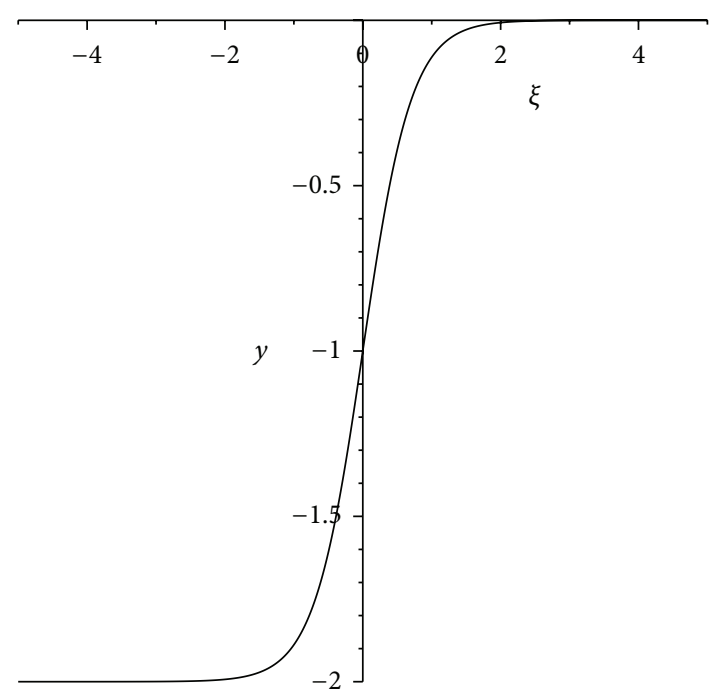

(b) $w_{4}(\xi)=\sqrt{k c}\left(-1+\tanh \left(\sqrt{2 k c} \xi / 2 k^{2}\right)\right) / 2 k$

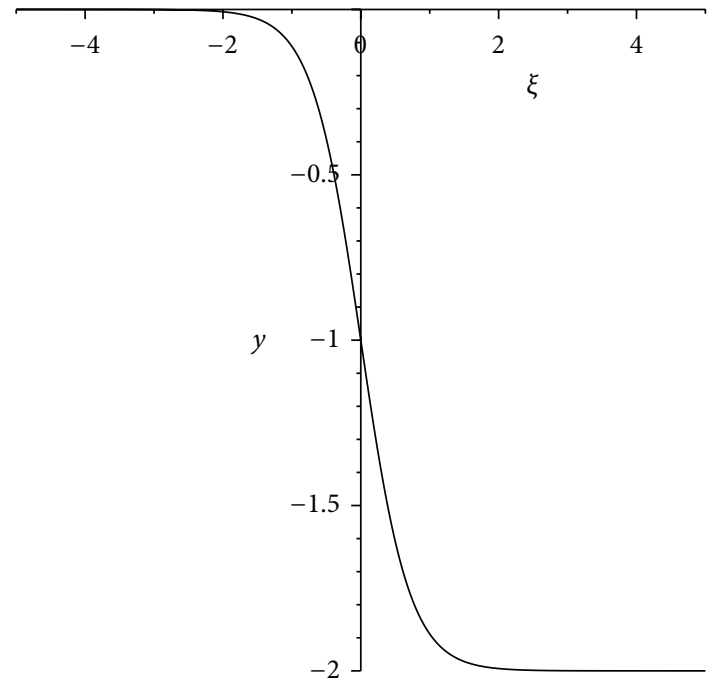

(d) $w_{4^{\prime}}(\xi)=\sqrt{k c}\left(1+\tanh \left(\sqrt{2 k c} \xi / 2 k^{2}\right)\right) /-2 k$

FIGURE 7: The kink (antikink) wave solutions of the system (11) and the system (3) when $r=-2 \sqrt{k c}, k>0, c>0$, and $\Delta \geq 0$.

Using the potential (4), the kink wave solution for the system (3) are obtained as follows:

$$
w_{4}(x, y, t)=-\frac{\sqrt{k c}\left(1-\tanh \left(\sqrt{2 k c}(k x+r y-c t) / 2 k^{2}\right)\right)}{2 k},
$$

where $w_{4}(x, y, t)$ is the smooth kink wave solution of the system (3). The smooth kink wave solution $w_{4}(\xi)$ of the system (3) is obtained from (51) (see Figure 7(b)). Analogously, completing the above integral (46b), we have the following antikink wave solution

$$
\varphi_{4^{\prime}}(\xi)=-\frac{\sqrt{k c}\left(1+\tanh \left(\sqrt{2 k c} \xi / 2 k^{2}\right)\right)}{2 k^{2}},
$$

$w_{4^{\prime}}(x, y, t)=-\frac{\sqrt{k c}\left(1+\tanh \left(\sqrt{2 k c}(k x+r y-c t) / 2 k^{2}\right)\right)}{2 k}$,

for the system (11) and the system (3), respectively (see Figures $7(\mathrm{c})$ and $7(\mathrm{~d}))$.

When $r=2 \sqrt{k c}$ (see Figure 2(i)), in $(\varphi, y)$-plane, the expressions of the heteroclinic orbits $\Gamma_{13}$ and $\Gamma_{14}$ passing the points $(0,0),\left(\varphi_{14}, 0\right)$ are given as

$$
y= \pm \sqrt{\frac{c \varphi^{2}}{k^{3}}-2 \frac{\sqrt{k c}}{k^{2}} \varphi^{3}+\frac{1}{2} \varphi^{4}}
$$

when $H(\varphi, y)=0, \varphi_{14}=\sqrt{k c} / k^{2}>0$. 
Substituting (54) into $d \varphi / d \xi=y$ and integrating them along $\Gamma_{13}$ and $\Gamma_{14}$, we have

$$
\begin{gathered}
\int_{\varphi}^{\varphi_{14}} \frac{\sqrt{2}}{\sqrt{\varphi^{2}\left(\varphi-\sqrt{k c} / k^{2}\right)^{2}}} d s=\int_{0}^{\xi} d s \\
-\int_{\varphi}^{\varphi_{14}} \frac{\sqrt{2}}{\sqrt{\varphi^{2}\left(\varphi-\sqrt{k c} / k^{2}\right)^{2}}} d s=\int_{0}^{\xi} d s .
\end{gathered}
$$

Completing the above integral (55a), we obtain the exact kink wave solution of the system (11) (see Figure 8(a)):

$$
\varphi_{5}(\xi)=\frac{\sqrt{k c}\left(1+\tanh \left(\sqrt{2 k c} \xi / 2 k^{2}\right)\right)}{2 k^{2}} .
$$

Noting (6), we obtain the following exact wave solution of the system (11) from (56):

$$
\begin{aligned}
q_{5}(x, y, t) & \\
= & \frac{\sqrt{k c}(1+\tanh (\sqrt{k c}(k x+r y-c t) / 2 k))}{2 k^{2}},
\end{aligned}
$$

where $q_{5}(x, y, t)$ is a kink wave solution of the system (11).

Since $u(\xi)=\int \varphi(\xi) d \xi$, integrating (56) about $\xi$, we can obtain

$$
\begin{aligned}
u_{5}(\xi)=\left(\frac{\sqrt{k c}}{2 k^{2}} \xi\right. & -\frac{\sqrt{2}}{4} \\
& \times\left(\ln \left(\tanh \left(\frac{\sqrt{k c} \xi}{2 k}\right)-1\right)\right. \\
& \left.\left.-\frac{\sqrt{2}}{4} \ln \left(1+\tanh \left(\frac{\sqrt{2 k c} \xi}{2 k^{2}}\right)\right)\right)\right) .
\end{aligned}
$$

According to (6), the traveling wave solution of (5) is able to obtain

$$
\begin{aligned}
& u_{5}(x, y, t) \\
& =\left(\frac{\sqrt{k c}}{2 k^{2}}(k x+r y-c t)\right. \\
& \quad-\frac{\sqrt{2}}{4}\left(\ln \left(\tanh \left(\frac{\sqrt{k c}(k x+r y-c t)}{2 k}\right)-1\right)\right. \\
& \left.\left.\quad-\frac{\sqrt{2}}{4} \ln \left(1+\tanh \left(\frac{\sqrt{2 k c}(k x+r y-c t)}{2 k^{2}}\right)\right)\right)\right) .
\end{aligned}
$$

Applying the potential (4), the kink wave solution of the system (3) is obtained as follows:

$$
\begin{aligned}
w_{5}(x, y, t) & \\
= & \frac{\sqrt{k c}(1+\tanh (\sqrt{k c}(k x+r y-c t) / 2 k))}{2 k},
\end{aligned}
$$

where $w_{5}(x, y, t)$ is the smooth kink wave solution of the system (3).

Noting (6), we can obtain the smooth kink wave solution $w_{5}(\xi)$ of the system (3) (see Figure 8(b)). Analogously, completing the above integral (55b), we obtain the exact antikink wave solution:

$$
\begin{aligned}
\varphi_{5^{\prime}}(\xi) & =\frac{\sqrt{k c}\left(1-\tanh \left(\sqrt{2 k c} \xi / 2 k^{2}\right)\right)}{2 k^{2}}, \\
w_{5^{\prime}}(\xi) & =\frac{\sqrt{k c}(1-\tanh (\sqrt{k c}(\xi) / 2 k))}{2 k},
\end{aligned}
$$

for the system (11) and the system (3), respectively (see Figures 8(c) and 8(d)).

Remark 7. In [3], applying the necessary condition for the kink waves to exist, multiple kink solutions and multiple singular kink solutions of the system (3) are formally derived. By the special traveling wave transform in [11], no kink (antikink) solutions of the system (11) and system (3) are obtained. In this paper, not considering the necessary condition for the kink waves to exist [3], we obtain all kink and antikink wave solutions of the system (11) and the system (3) by the bifurcation method of dynamical systems.

From (1) to (12), we can obtain three theorems about the exact periodic wave solutions and smooth solitary wave solutions and kink (antikink) wave solutions for the system (11) and the system (3).

Theorem 8. When conditions (I) or (II) hold, one can obtain some representative smooth exact periodic wave solutions of the system (11) from the periodic obits (see Figures 2(a), 2(c), 2(i), 2(j), and 2(l)) and Figures 3(a) and 3(b)), which correspond to the representative smooth exact periodic wave solutions of the system (3), such as the periodic wave solution $q_{1}(x, y, t)$ of the system (11) corresponding to the periodic wave solution $w_{1}(x, y, t)$ of the system (3).

Theorem 9. Under conditions (I), the following results hold.

(1) When $-4 \sqrt{3 k c} / 3<r<-2 \sqrt{k c}$, the system (11) has an exact solitary wave solution $q_{2}(x, y, t)$, which corresponds to the solitary wave solutions $w_{2}(x, y, t)$ of the system (3).

(2) When $2 \sqrt{k c}<r<4 \sqrt{3 k c} / 3$, the system (11) has exact solitary wave solutions $q_{3}(x, y, t)$, which correspond to the solitary wave solutions $w_{3}(x, y, t)$ of the system (3).

Theorem 10. Under conditions (I), the following results hold.

(1) When $r=-2 \sqrt{k c}$, the system (11) has an exact smooth kink wave solution $q_{4}(x, y, t)$, which corresponds to 


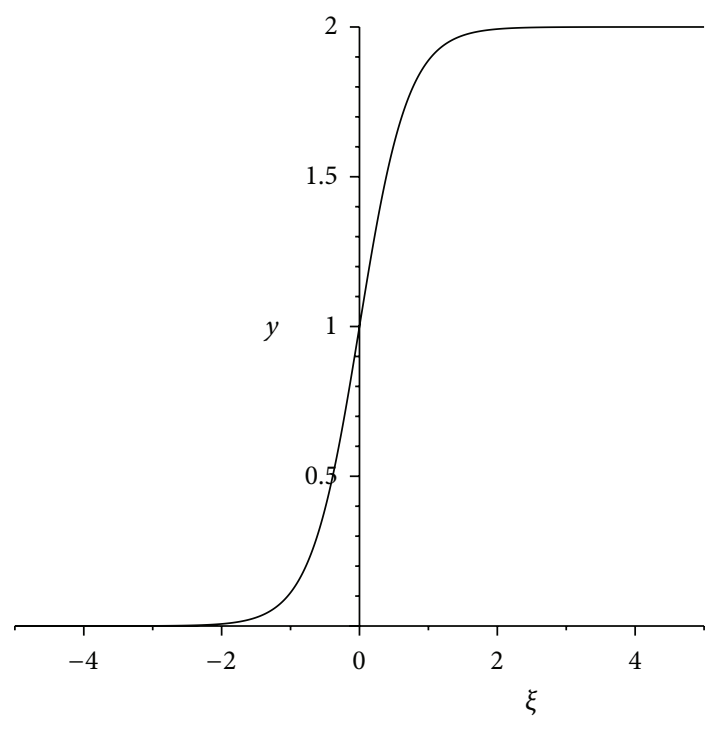

(a) $\varphi_{5}(\xi)=\sqrt{k c}\left(1+\tanh \left(\sqrt{2 k c} \xi / 2 k^{2}\right)\right) / 2 k^{2}$

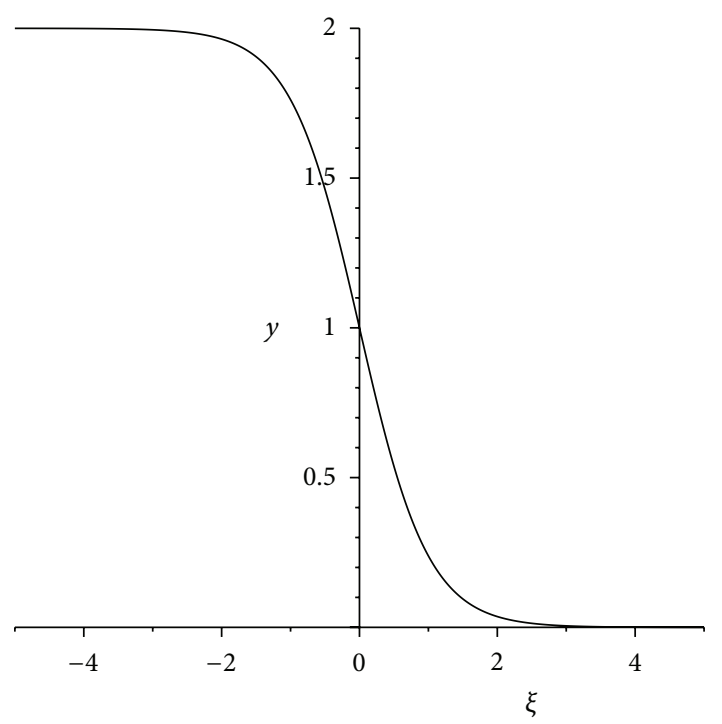

(c) $\varphi_{5^{\prime}}(\xi)=\sqrt{k c}\left(1-\tanh \left(\sqrt{2 k c} \xi / 2 k^{2}\right)\right) / 2 k^{2}$

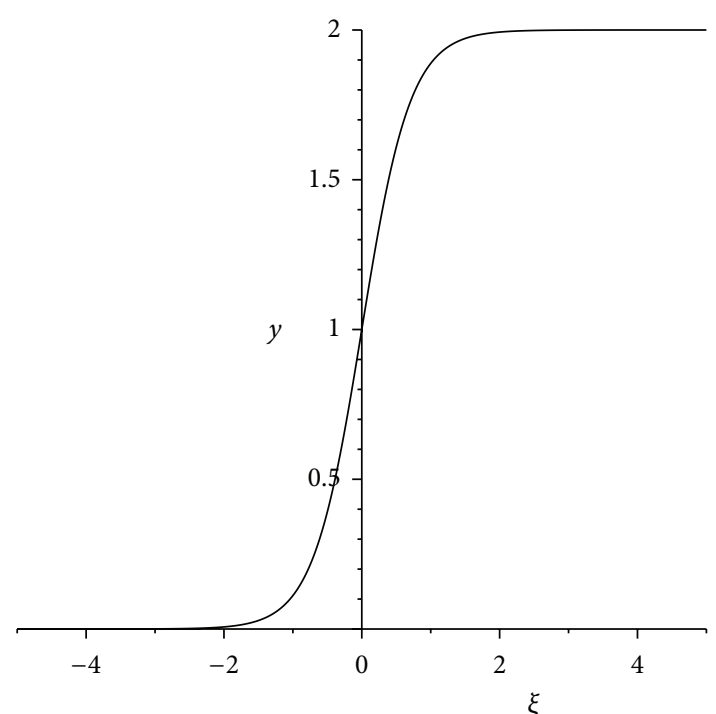

(b) $w_{5}(\xi)=\sqrt{k c}(1+\tanh (\sqrt{k c} \xi / 2 k)) / 2 k$

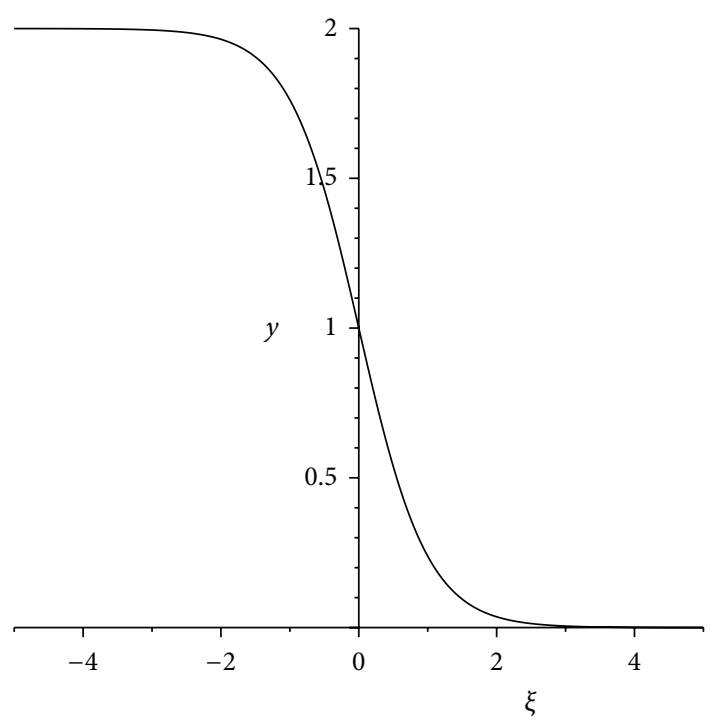

(d) $w_{5^{\prime}}(\xi)=\sqrt{k c}(1-\tanh (\sqrt{k c} \xi / 2 k)) / 2 k$

FIGURE 8: The kink (antikink) wave solutions of system (11) and (3) when $r=2 \sqrt{k c}, k>0, c>0$, and $\Delta \geq 0$.

the kink wave solution $w_{4}(x, y, t)$ of the system (3), respectively.

(2) When $r=2 \sqrt{k c}$, the system (11) has an exact smooth kink wave solution $q_{5}(x, y, t)$, which corresponds to the kink wave solution $w_{5}(x, y, t)$ of the system (3).

\section{Discussion}

In this work we obtain all wave solutions of the complete integrability of the $(2+1)$-dimensional nonlinear evolution equation, the third model, by dynamical systems method. This method can be used for the remaining three models. By determining the necessary condition for the complete integrability of these models in [3], multiple kink solutions and multiple singular kink solutions were formally derived for the third model. Compared to the method in [3], the necessary condition which is among the coefficients of the spatial variables is not necessary in our method. In [11], only solitary wave solutions are obtained by a special traveling wave transform. To our knowledge, we completely obtain all solitary wave solutions and kink (antikink) wave solutions for these models by the bifurcation method of dynamical system. The idea of applying the method of dynamical system to research the complete integrability can be used for other models. This will be examined in forthcoming works. For the remaining three models, we can follow the same approach to derive all wave solutions for them. 


\section{Conflict of Interests}

The authors declare that there is no conflict of interests regarding the publication of this paper.

\section{Acknowledgments}

The authors gratefully acknowledge the support of the National Natural Science Foundation of China through Grant nos. 11072007, 11372014, and 11290152, the Natural Science Foundation of Beijing through Grant no. 1122001, the International Science and Technology Cooperation Program of China through Grant no. 2014DFR61080, and the Research Fund for the Doctoral Program of Higher Education of China through Grant no. 20131103120027. All authors wish to thank professor Li Jibin for many valuable suggestions leading to the improvement of this paper.

\section{References}

[1] X. Geng, C. Cao, and H. H. Dai, "Quasi-periodic solutions for some $(2+1)$-dimensional integrable models generated by the Jaulent-Miodek hierarchy," Journal of Physics A: Mathematical and General, vol. 34, no. 5, pp. 989-1004, 2001.

[2] X. Geng and Y. Ma, "N-soliton solution and its Wronskian form of a (3+1)-dimensional nonlinear evolution equation," Physics Letters A, vol. 369, no. 4, pp. 285-289, 2007.

[3] A. Wazwaz, "Multiple kink solutions and multiple singular kink solutions for $(2+1)$-dimensional nonlinear models generated by the Jaulent-Miodek hierarchy," Physics Letters A, vol. 373, no. 21, pp. 1844-1846, 2009.

[4] Y. Y. Zhang, X. Q. Liu, and G. W. Wang, "Symmetry reductions and exact solutions of the $(2+1)$-dimensional Jaulent-Miodek equation," Applied Mathematics and Computation, vol. 219, no. 3, pp. 911-916, 2012.

[5] R. Hirota, The Direct Method in Soliton Theory, vol. 155, Cambridge University Press, Cambridge, UK, 2004.

[6] W. Hereman and A. Nuseir, "Symbolic methods to construct exact solutions of nonlinear partial differential equations," Mathematics and Computers in Simulation, vol. 43, no. 1, pp. 1327, 1997.

[7] A. M. Wazwaz, Partial Differential Equations and Solitary Waves Theorem, Springer and HEP, Berlin, Germany, 2009.

[8] A. Wazwaz, "Multiple soliton solutions for some $(3+1)$-dimensional nonlinear models generated by the Jaulent-Miodek hierarchy," Applied Mathematics Letters, vol. 25, no. 11, pp. 19361940, 2012.

[9] J. B. Li and G. R. Chen, "Exact traveling wave solutions and their bifurcations for the Kudryashov-Sinelshchikov equation," International Journal of Bifurcation and Chaos, vol. 22, no. 5, Article ID 1250118, pp. 1-19, 2012.

[10] J. Li, Y. Zhang, and X. Zhao, "On a class of singular nonlinear traveling wave equations (II): an example of Gckdv equations," International Journal of Bifurcation and Chaos in Applied Sciences and Engineering, vol. 19, no. 6, pp. 1995-2007, 2009.

[11] H. Liu and F. Yan, "The bifurcation and exact travelling wave solutions for $(2+1)$-dimensional nonlinear models generated by the Jaulent-Miodek hierarchy," International Journal of Nonlinear Science, vol. 11, no. 2, pp. 200-205, 2011.
[12] Y. Zhou and Q. Liu, "Bifurcation of travelling wave solutions for a $(2+1)$-dimensional nonlinear dispersive long wave equation," Applied Mathematics and Computation, vol. 189, no. 1, pp. 970979, 2007. 


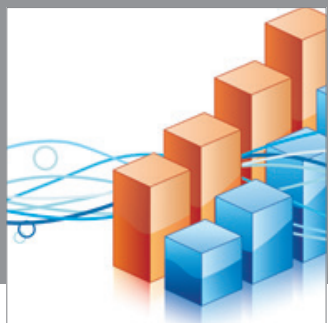

Advances in

Operations Research

mansans

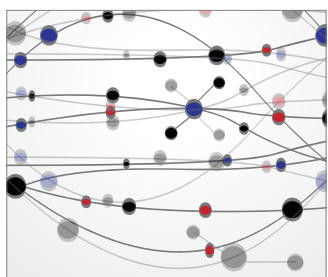

The Scientific World Journal
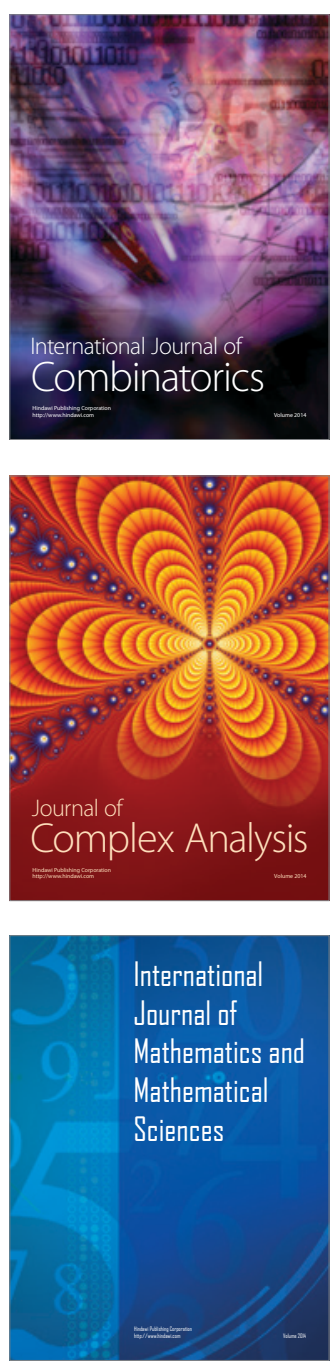
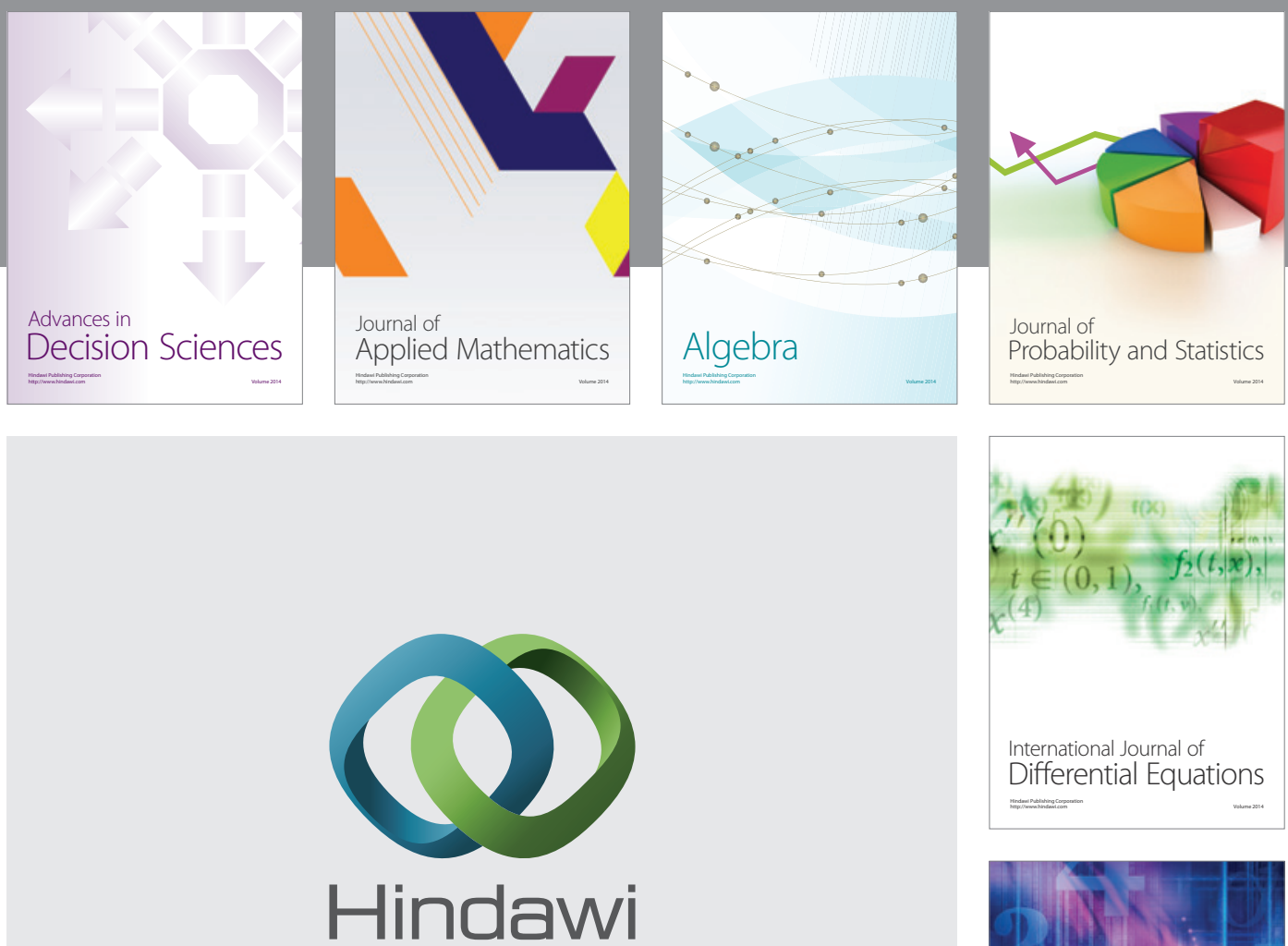

Submit your manuscripts at http://www.hindawi.com
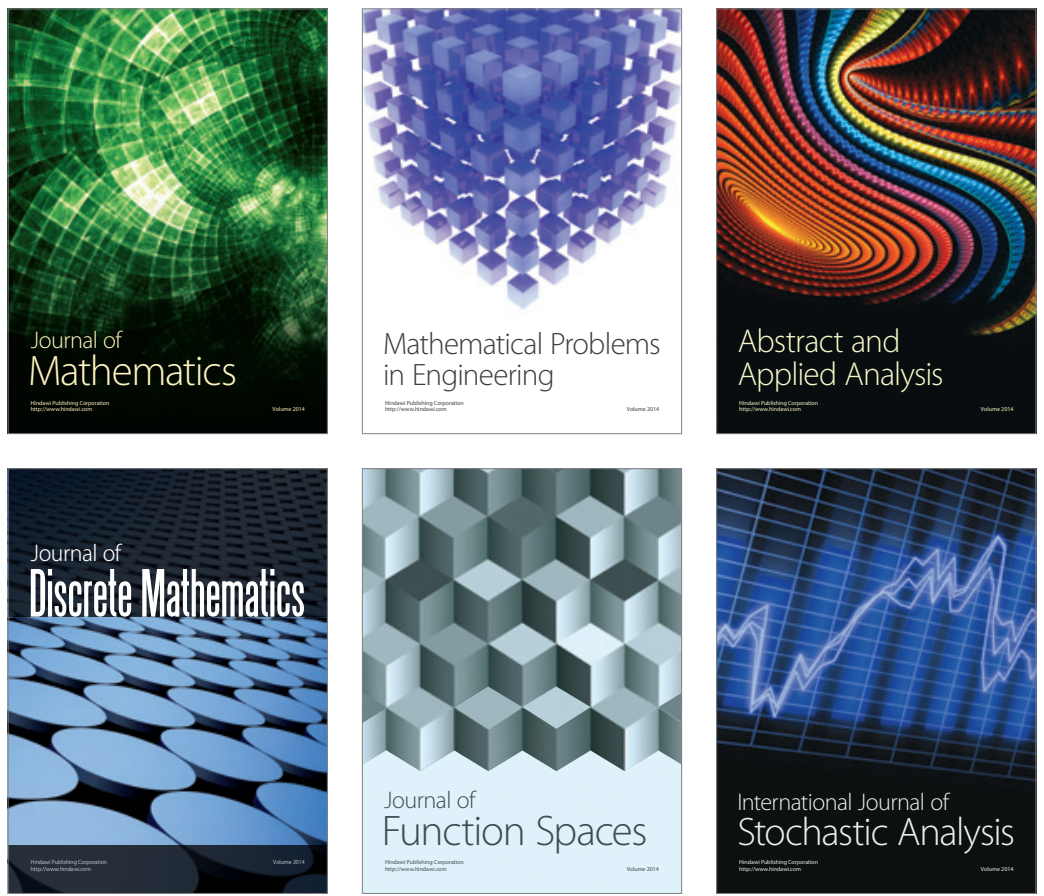

Journal of

Function Spaces

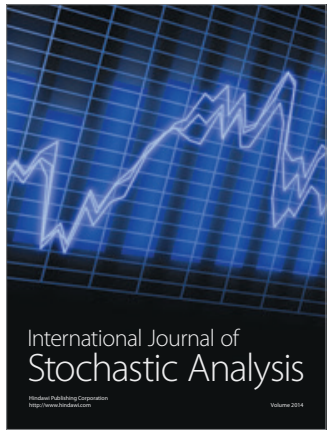

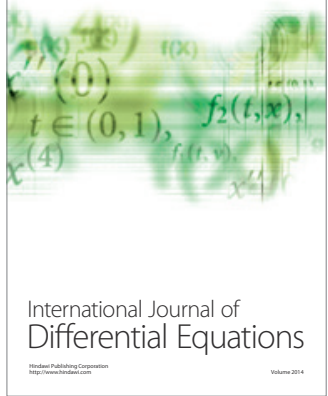
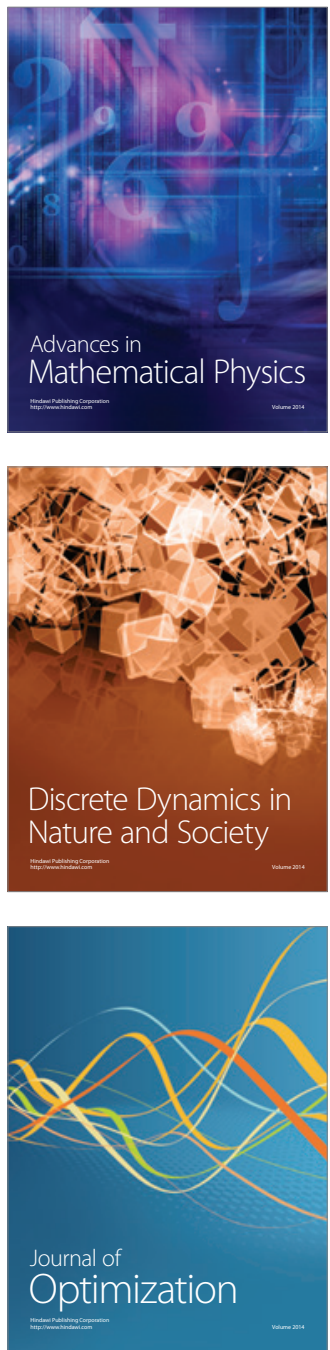